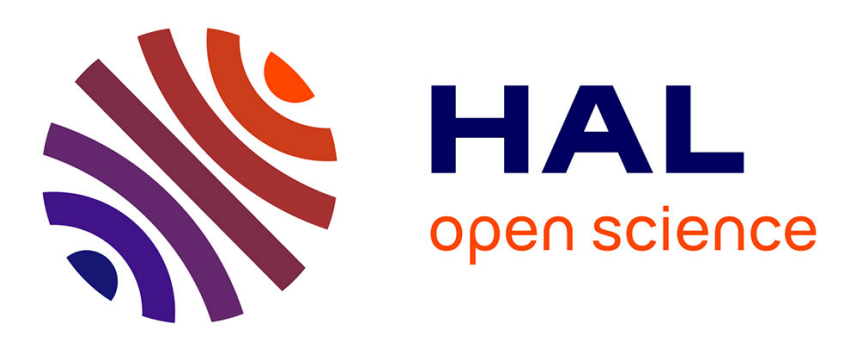

\title{
Effect of an external excitation on the flow structures in a circular impinging jet
}

Jiří Vejražka, J. Tihon, Philippe Marty, V. Sobolik

\section{To cite this version:}

Jiří Vejražka, J. Tihon, Philippe Marty, V. Sobolik. Effect of an external excitation on the flow structures in a circular impinging jet. Physics of Fluids, 2005, 17 (10), 10.1063/1.2084207 . hal00265718

\section{HAL Id: hal-00265718 \\ https://hal.science/hal-00265718}

Submitted on 5 Feb 2020

HAL is a multi-disciplinary open access archive for the deposit and dissemination of scientific research documents, whether they are published or not. The documents may come from teaching and research institutions in France or abroad, or from public or private research centers.
L'archive ouverte pluridisciplinaire HAL, est destinée au dépôt et à la diffusion de documents scientifiques de niveau recherche, publiés ou non, émanant des établissements d'enseignement et de recherche français ou étrangers, des laboratoires publics ou privés. 


\title{
Effect of an external excitation on the flow structure in a circular impinging jet
}

\author{
J. Vejrazka and J. Tihon ${ }^{\text {a) }}$ \\ Institute of Chemical Process Fundamentals, Academy of Sciences of the Czech Republic, Rozvojová 135, \\ 16502 Prague 6, Czech Republic \\ Ph. Marty \\ LEGI-GRETh, CEA-Grenoble, 17 Av. des Martyrs, 38054 Grenoble Cedex 9, France \\ V. Sobolík \\ LMTAI, av. Michel Crepeaux, 17042 La Rochelle, France
}

(Received 8 March 2005; accepted 20 July 2005; published online 11 October 2005)

\begin{abstract}
The effect of an external excitation on circular impinging jet flow is studied experimentally. Velocity fields in moderate air flows $(\mathrm{Re}=10000)$ are investigated by hot-wire anemometry. The basic flow is excited by a small sinusoidal modulation of the nozzle exit velocity. The phase-averaging technique is used to study the behavior of vortex structures in the jet, specifically rolling-up, pairing, and interaction with the wall. The jet is found to be sensitive to excitation in the frequency range characterized by a Strouhal number, $\mathrm{St}_{e}=f_{e} D / U$, from 0.3 to 3 . Different flow regimes are identified in the excited impinging jet: a periodic flow regime with the same frequency as the excitation, a regime with a frequency corresponding to a subharmonic of the excitation frequency, a regime alternating between these two frequencies, and two border regimes with more complicated behavior. The low-frequency excitation leads to the formation of vortices, which are larger than those occurring in an unexcited jet. Consequently, the near-wall velocity fluctuations are enhanced and the unsteady flow separation induced by vortex impingement is more pronounced. By contrast, excitation at higher frequencies, characterized by value 0.017 of a Strouhal number based on the shear layer thickness $\theta, \mathrm{St}_{\theta}=f_{e} \theta / U$, leads to the roll-up of small vortices and hence to the attenuation of the near-wall velocity fluctuations. In this case, the flow separation is suppressed because the small vortices are unable to induce it. Finally, the vortex merging process is sensitive to the double-frequency excitation with subharmonic components. For example, a combined excitation at $\mathrm{St}_{e}=1.60$ and 0.53 leads to the merging of three vortices. (C) 2005 American Institute
\end{abstract} of Physics. [DOI: 10.1063/1.2084207]

\section{INTRODUCTION}

Jet impingement is used in systems where high heat transfer rate between the wall and the fluid is required. ${ }^{1}$ Three characteristic zones can be distinguished in the impinging jet flow (see Fig. 1): (i) the jet zone, where the flow does not feel the presence of the wall and it is therefore similar to free jet flow; (ii) the stagnation zone, where the wall imposes pressure gradients which deviate the flow from the axial to the radial direction; and (iii) the wall jet zone, where the mean flow is more or less parallel to the wall.

Free jets and their instabilities have been extensively studied. ${ }^{2-9}$ The jet flow at moderate Reynolds numbers is dominated by large vortices, which emerge as result of convective instability of the shear layer near the nozzle lip. This instability is controlled by the processes taking place at two different length scales. The first length scale is the momentum thickness $\theta$ of the boundary layer at the nozzle lip and is important for the initial development of the shear layer. The second length scale is the jet diameter $D$, which becomes important further downstream. The shear layer near the

\footnotetext{
${ }^{a}$ Author to whom correspondence should be addressed. Electronic mail: tihon@icpf.cas.cz
}

nozzle lip is inviscidly unstable to both the axisymmetric ${ }^{2}$ and helical instability modes. ${ }^{3-5}$ These two modes have comparable amplification rates and their frequencies differ only slightly. However, they do not appear at the same time as the jet switches between them. ${ }^{5}$

Near the nozzle, the mode with the highest theoretical growth rate is the axisymmetric mode, whose frequency $f$ is characterized by the Strouhal number $\mathrm{St}_{\theta}$ $=f \theta / U \sim 0.017 .{ }^{2,10,11}$ However, the dominant frequency observed in this region is lower, about $\mathrm{St}_{\theta}=0.012 .^{5,11,12}$ The shift in the dominant frequency can be explained by feedback from the downstream flow events. The vortex rolling-up process, which results from the saturation of shear layer waves, induces pressure waves affecting back the flow at the nozzle lip. Such a feedback loop enhances the flow fluctuations at the modified dominant frequency. ${ }^{12}$

Before the saturation, the waves become unstable to an instability mode with the subharmonic frequency. ${ }^{12,13}$ The growth of this subharmonic mode then leads to vortex pairing. ${ }^{10,13}$ More than one successive pairing may occur and the vortices with the initial length scale $\theta$ grow to match the final length scale $D$. The prevailing frequency at the end of the potential core then ranges from $\mathrm{St}=f D / U=0.3$ to $0.5{ }^{6}$ 


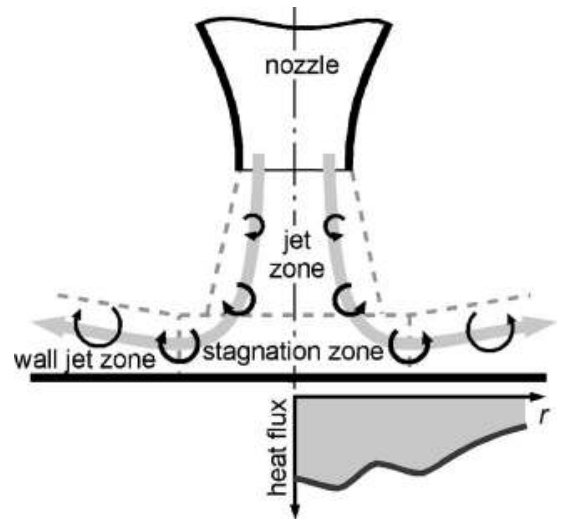

FIG. 1. Characteristic zones of the flow in an impinging jet.

depending on the configuration of experimental setup as well as on the initial shear layer thickness. ${ }^{10}$ At the same time the fluid jet loses its axial symmetry and streamwise vortices appear. This process was studied experimentally by Liepman and Gharib $^{7}$ and numerically by Brancher et al. ${ }^{8}$

The vortex formation and subsequent evolution of the coherent structures in free jets can be controlled by the flow excitation. ${ }^{9-11,14-17}$ The influence of the excitation frequency, $f_{e}$, and the amplitude on the flow behavior of vortex structures was documented, e.g., by Zaman and Hussain ${ }^{14}$ or Broze and Hussain. ${ }^{15}$ A remarkable flow response is observed at the excitation frequency of $\mathrm{St}_{e, \theta}=f_{e} \theta / U=0.017$, when relatively small but stable vortices are formed and flow turbulence is attenuated. ${ }^{11}$ This excitation promotes the growth of instability modes, which do not appear in natural (i.e., unexcited) jets due to their weak feedback (and selfexcitation) at the nozzle lip. ${ }^{12}$

Similar vortex structure is also observed in the impinging jets. ${ }^{18-20}$ When a temperature difference exists between the fluid and the wall, the vortex-wall interaction is expected to influence the heat transfer. For small nozzle-to-plate distances, $H \leqslant 4 D$, the profile of local heat transfer rates (schematized in Fig. 1) exhibits two distinct peaks. ${ }^{21,22}$ The position of the local heat transfer minimum between these two peaks corresponds to the place where the vortices touch the wall. The wall shear stresses also exhibit a local minimum in this region. ${ }^{23}$ According to Liu and Sullivan, ${ }^{24}$ the boundary layer separation is responsible for this local decrease in transfer rates. This unsteady separation of the boundary layer $^{25,26}$ is induced by an unfavorable pressure gradient, which is generated in front of the vortex due to its impact on the wall. The flow separation brings about pressure waves, ${ }^{27,28}$ which also have a feedback effect on the initial shear layer instability.

As in the case of free jets, the vortex behavior of impinging jets can be modified by the flow excitation. This raises the question of how such a modification of the flow character affects the wall transfer processes. In order to answer this question, Liu and Sullivan ${ }^{24}$ examined the excited impinging jet with a small nozzle-to-plate spacing $(H / D$ $\leqslant 2$ ). They found that excitation at a frequency of $\mathrm{St}_{e}$ $=f_{e} D / U=0.85$, which creates strong well-organized vortices impinging onto the wall, leads to a local reduction of wall heat transfer. By contrast, they achieved a local heat transfer enhancement at the high-frequency excitation of $\mathrm{St}_{e}=1.6$, which produces a random structure of small-scale irregular vortices. Hwang and $\mathrm{Cho}^{22}$ also showed that the flow excitation modifies the wall heat transfer in the impinging jet, especially in the wall jet region at radii, $r$, between $0.75 D$ and $2.5 \mathrm{D}$. Alekseenko et $a l^{23}$ used electrochemical friction probes to measure the wall shear stress distribution in the impinging jet at moderate and low Reynolds numbers. Significant drag reduction, growth of fluctuation level, enhancement or suppression of broadband turbulence was observed depending on the flow excitation conditions.

Although there is basic knowledge of excited impinging jets, the data documenting the effect of excitation on flow behavior and heat/mass transfer are rather scarce. The previous experimental works have largely been carried out at limited excitation conditions and a more comprehensive experimental study is still lacking. The aim of this article is to document the effect of flow excitation on the entire velocity field in a round impinging jet. First, the hot-wire anemometry was used to measure the local near-wall flow response to jet excitation over a wide frequency range. Then, the velocity field was examined in detail at several excitation frequencies. To demonstrate the flow behavior of large vortex structures, a phase-averaging technique was applied to the periodic velocity data. As the experiments were time-consuming, our research was limited to a single combination of Reynolds number and nozzle-to-plate distance $(\operatorname{Re}=10000, H / D=2)$.

\section{DESCRIPTION OF THE EXPERIMENT}

\section{A. Experimental setup and measurement technique}

The experimental setup ${ }^{29}$ designed for the velocity field measurements is shown in Fig. 2. Air from a high pressure circuit was filtered then supplied to a settling chamber through two successive pressure-reduction valves, a needle valve and a rotameter. The settling chamber, with a diameter of $180 \mathrm{~mm}$ and a length of $650 \mathrm{~mm}$, was assembled from nine elements of a PVC tube. A pressure tap and a temperature sensor were inserted into the last element of the settling chamber. The rear part of the settling chamber was equipped with a midtone loudspeaker $(8 \Omega, 20 \mathrm{~W})$, which was used to induce velocity pulsations in the flow. The movement of the loudspeaker membrane was controlled by a sinusoidal voltage signal generated by a digital-analog converter and amplified by a $50 \mathrm{~W}$ amplifier. The air passed through four grids and a honeycomb mounted into the settling chamber and formed a jet after going through a bell-shaped round nozzle. The shape of the plastic nozzle was prescribed by a fourth-order polynomial and the nozzle exit diameter was $D=25 \mathrm{~mm}$. The large contraction ratio of the nozzle ensured a flat exit velocity profile with a minimal turbulence. The Plexiglas impingement plate $(600 \times 600 \mathrm{~mm})$ was fixed at a distance of $H=50 \mathrm{~mm}$ from the nozzle lip. The central part of this square plate was removed and replaced by an aluminum plate $(300 \times 150 \mathrm{~mm})$ for future temperature field measurement.

Two single hot-wire probes were used to measure the absolute value of the velocity at different locations. A bent 


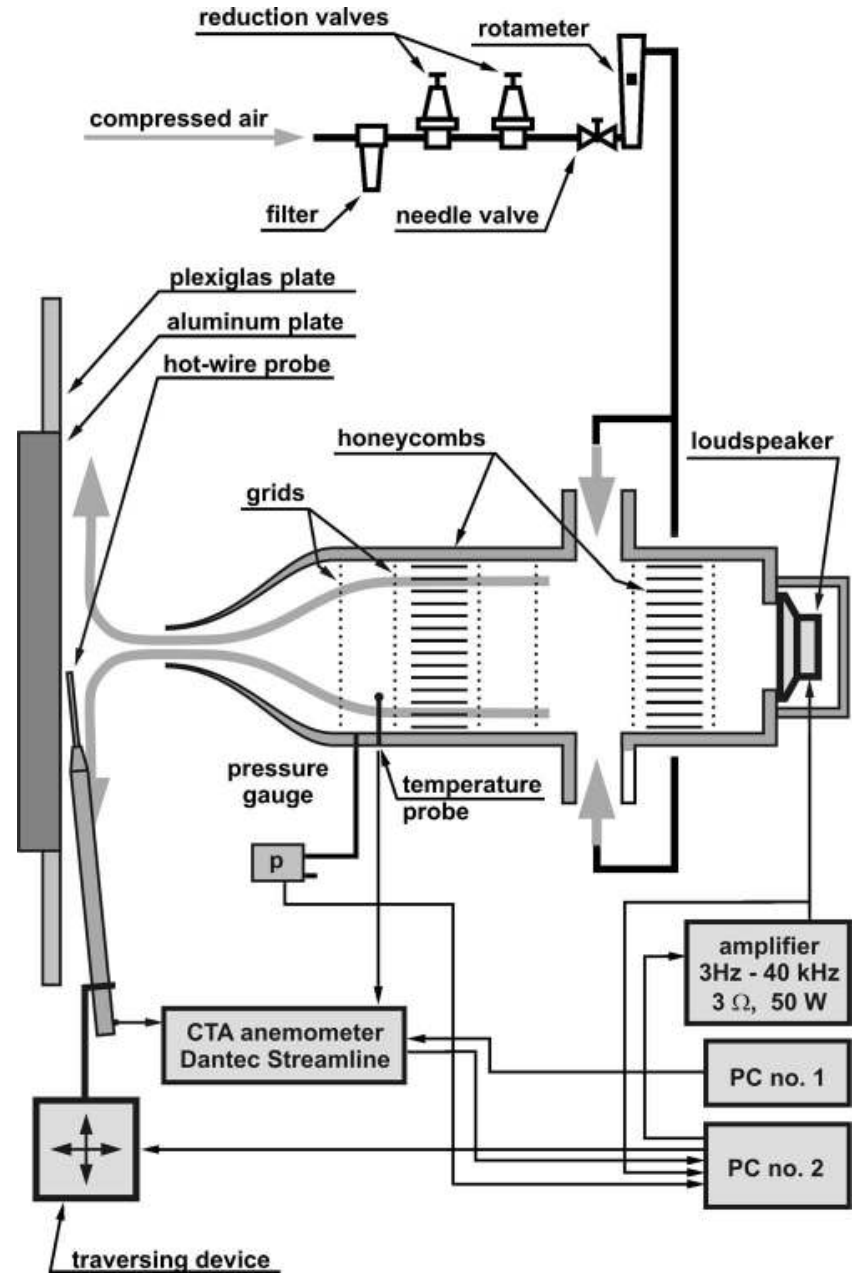

FIG. 2. Experimental setup.

probe Dantec 55P14 was used for measurements in the jet zone and a straight probe Dantec 55P11 in the wall zone. The active element of both probes is a platinum-coated tungsten wire, which has a diameter of $5 \mu \mathrm{m}$ and a length of $1.25 \mathrm{~mm}$. The probes were operated as velocity sensors using a constant-temperature module of a Dantec Streamline anemometer. To precisely adjust the measuring position, the probes were mounted on a $2 \mathrm{D}$ traversing device, which provided a displacement precision of $25 \mu \mathrm{m}$. The pressure and temperature inside the settling chamber were measured by a pressure transducer and a temperature probe of the anemometer, respectively. Two computers controlled the measuring conditions, data acquisition and processing. National Instruments hardware and custom software were used to control the traversing device, the loudspeaker, and the data acquisition.

\section{B. Experimental procedure, calibrations, and data treatment}

\section{Experimental procedure}

To measure the velocity field inside the impinging jet, the probe was moved by the traversing device to a preset point of the measuring grid. The anemometer, pressure transducer, thermometer and loudspeaker provided four measured
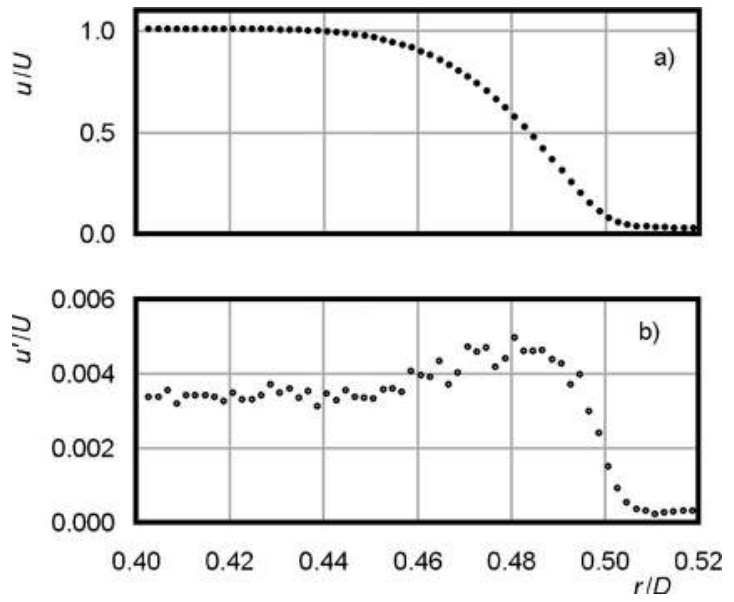

FIG. 3. Velocity profiles in close proximity to the nozzle exit: (a) normalized time-average velocity and (b) velocity rms.

voltage signals. For each measuring point, $4 \times 60000$ data samples were acquired at a sampling frequency of $12 \mathrm{kHz}$. The experiments were limited to one combination of the Reynolds number $\operatorname{Re}=D U / \nu=10000$ (for air viscosity of $\nu=15.25 \times 10^{-6} \mathrm{~m}^{2} \mathrm{~s}^{-1}$ and an average nozzle velocity of $U=6.1 \mathrm{~m} \mathrm{~s}^{-1}$ ) and the nozzle-to-plate distance $H / D=2$. The excitation frequency $f_{e}$ was varied from 50 to $1000 \mathrm{~Hz}$ (corresponding to an excitation Strouhal number $\mathrm{St}_{e}$ ranging from 0.2 to 4 ). The amplitude of flow perturbations at the nozzle exit was maintained at a level of $u=0.01 U$. To keep this level constant for all excitation frequencies, the amplitude of the voltage signal supplying the loudspeaker was adjusted. All measurements were carried out at a temperature of about $22{ }^{\circ} \mathrm{C}$.

\section{Nozzle velocity profile}

The nozzle velocity profile measured for $\mathrm{Re}=10000$ at a distance $0.5 \mathrm{~mm}$ downstream from the nozzle lip is presented in Fig. 3. The hat-shaped velocity profile was slightly dimpled around the centerline. The velocity difference between the local maximum value observed near the nozzle lip and the centerline value was about $1 \%$. The boundary layer has a convectional thickness, $\delta_{0.95}$, of $1.19 \mathrm{~mm}$, a displacement thickness, $\delta^{*}$, of $0.49 \mathrm{~mm}$ and a momentum thickness, $\theta$, of $0.21 \mathrm{~mm}$. The turbulence intensity reached $0.35 \%$ inside the flat velocity profile and increased to $0.5 \%$ inside the boundary layer.

\section{Calibration of the hot-wire probes}

The flat velocity profile at the nozzle exit was used to calibrate the hot-wire probes. The probe was placed at the nozzle centerline and the flow rate was adjusted with the needle valve. The actual flow velocity was calculated from the measured value of the chamber pressure using the Bernoulli equation. The Collins-Williams formula, relating the wire Reynolds number, $\mathrm{Re}_{w}$, and the wire Nusselt number, $\mathrm{Nu}_{w}$, was applied to fit the calibration data: 


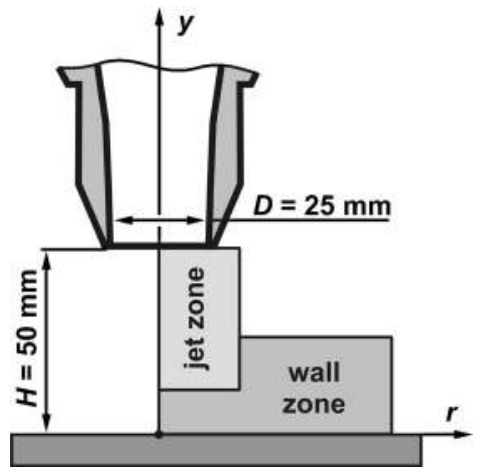

FIG. 4. Coordinate system for the impinging jet and measuring zones.

$$
\mathrm{Nu}_{w}\left(\frac{T_{w}+T_{\mathrm{amb}}}{2 T_{\mathrm{amb}}}\right)^{M}=A+B \cdot \mathrm{Re}_{w}^{N} .
$$

To obtain a good fit of the four constants $(A, B, M$, and $N)$, each calibration was done for several wire temperatures, $T_{w}$, by varying the overheat ratio of the anemometer bridge at a given ambient temperature $T_{a m b}$. The probes were calibrated every six hours of operation.

\section{Measuring grid}

For the velocity field measurements, the measuring grid was divided into two zones shown in Fig. 4. The bent probe was used in the "jet zone" delimited by $y / D$ from 0.35 to 2 (nozzle exit) and $r / D$ from 0 (centerline) to 0.9 . The straight probe was applied in the "wall zone" region with $y / D$ from 0 (wall) to 1 and $r / D$ from 0 (centerline) to 2.65. The grid spacing was roughly $1 \mathrm{~mm}$, however a finer grid with a step of $0.2 \mathrm{~mm}$ was used for detailed near-wall measurements. In total, there were about 3500 measuring points. During measurements the probe was moved automatically through the measuring grid.

\section{Data processing}

Since the single hot-wire probes does not allow distinction between the wall-normal, $u_{y}$, and wall-parallel, $u_{r}$, velocity components, the measured quantity corresponds to a local value of velocity magnitude, $u=\left(u_{y}+u_{r}\right)^{1 / 2}$. Only in the "free jet" zone located close to the nozzle and in the wall jet zone formed after impingement, it is practically reduced to the single velocity components $u_{y}$ and $u_{r}$, respectively. Everywhere in-between, these velocity components are not separable. This limitation is also pronounced in the near-wall region within the unsteady separated flow, where the magnitude of both velocity components is of the same order. But still, the maps of time-averaged velocities, phase-averaged velocities and velocity fluctuations reconstructed during the post-processing of $u$ signals provide valuable information on behavior of flow structures inside the jet.

As the strong velocity fluctuations are appearing due to the passage of large coherent structures rather than to broadband turbulence, we prefer to avoid the use of the term "turbulence" or "turbulence intensity." We will use the term "velocity rms" for the root mean square value of velocity fluctuations normalized by the average nozzle velocity, $u^{\prime} / U$.

To calculate the power spectra of velocity fluctuations, the measured signals were divided into 50 overlapping parts each with 2048 samples. After subtracting the mean value and applying the Hanning windowing function, a standard fast Fourier transform (FFT) was applied to each part. The average spectrum, $G_{u u}$, was then normalized to meet the following relationship:

$$
\int_{0}^{\infty} G_{u u}(\mathrm{St}) d \mathrm{St}=\left(u^{\prime} / U\right)^{2} .
$$

The phase averaging technique (see, e.g., Hussain and Zaman $^{16}$ ) was used in this work to extract the periodic component from the velocity signals and to determine the phase relationships between the signals measured at different points. The sinusoidal voltage signal to the loudspeaker served as a phase reference. When the voltage increased above a threshold level, it triggered the reference time events, which divided the measured signals into the distinct phase periods. The phase-averaged velocities, $\langle u\rangle$, were calculated according to the following formula:

$$
\langle u\rangle_{k}=\frac{1}{N} \sum_{i=0}^{N-1} u_{k+i n}, \quad k=0,1, \ldots, n-1,
$$

where $n$ is the number of samples per period (usually between 30 and 70), $N$ is the number of periods (ranging from 700 to 3500 ), and the index $k$ indicates a particular phase. The simple relationship between the time-averaged velocity, $\bar{u}$, and phase-averaged velocity is obvious

$$
\bar{u}=\frac{1}{n} \sum_{k=0}^{n-1}\langle u\rangle_{k} \text {. }
$$

As the flow was periodic in some excitation cases, the phase-averaged velocity map obtained for the whole measuring domain could be interpreted as an instantaneous velocity field corresponding to a particular phase time. A sequence of such velocity maps could be animated to show the development of flow structures in the impinging jet flow. All velocity maps and movies obtained for the studied excitation conditions are available as the supplementary data at the EPAPS site. $^{30}$

\section{RESULTS AND DISCUSSION}

\section{A. Near-wall flow regimes induced by the jet excitation}

The flow in the near-wall region is sensitive to the jet excitation and depends strongly on the excitation frequency. This is demonstrated in Figs. 5 and 6, where time records and corresponding frequency spectra of the velocity signals are presented. During these measurements the hot-wire probe was placed at a fixed point $(r / D=1$ and $y / D=0.04)$ located near the wall at the radius, where the vortices impinge onto the wall. Under unexcited flow conditions [Fig. 5(a)], strong velocity fluctuations are observed due to irregular large-scale 

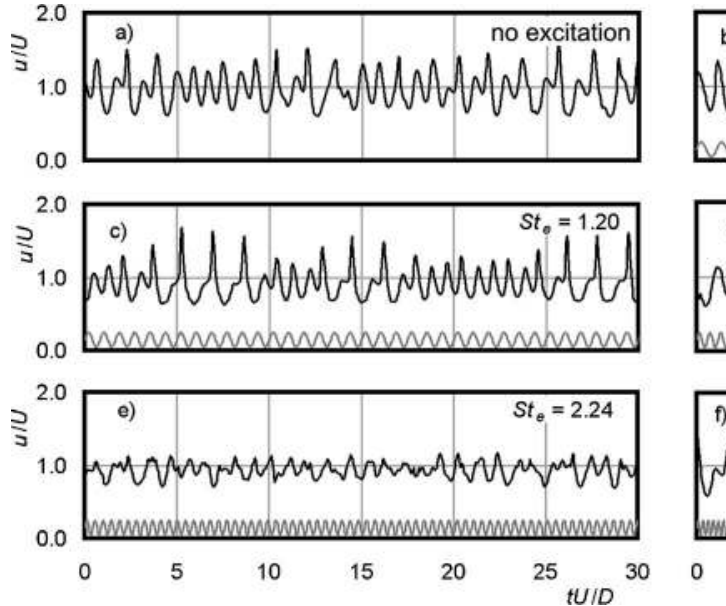
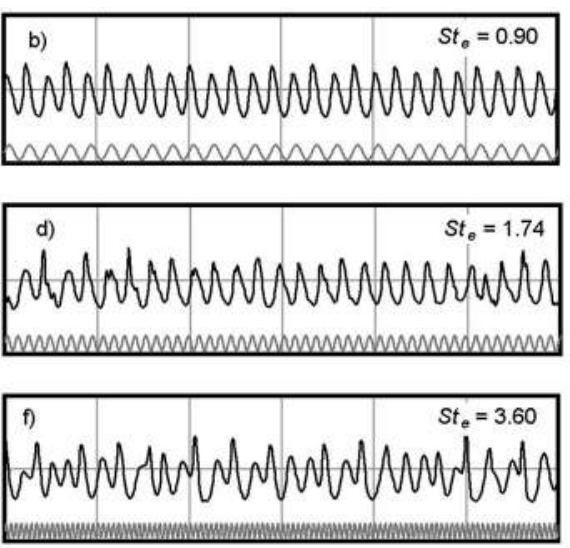

0
10
15
20
$25{ }_{t U / D}^{30}$
FIG. 5. Velocity signals measured in the near-wall region $(r / D=1, y / D$ $=0.04)$ at the different jet excitation frequencies. The sinusoidal excitation signals are shown at the bottom of each graph (excitation amplitude and phase are not to scale). vortices passing through the near-wall region. These fluctuations exhibit a wide-spectrum character [Fig. 6(a)], but the dimensionless frequency $\mathrm{St}=f D / U \sim 1$ still dominates the signal. Another peak around $\mathrm{St} \sim 0.5$ suggests vortex pairing. When the jet is excited at a low frequency, the measured velocity signal remains unaffected by the excitation applied at the nozzle. As soon as the excitation frequency reaches the value corresponding to an excitation Strouhal number of $\mathrm{St}_{e} \sim 0.3$, the amplitude of near-wall flow fluctuations starts to increase and at the same time the flow becomes more regular. If the excitation is kept in the range of frequencies present in the unexcited impinging jet ( $\mathrm{St}_{e}$ from 0.6 to 1.1), the near-wall flow has a periodic character [Fig. 5(b)], perhaps with a slight subharmonic modulation. This is reflected by appearance of a sharp peak in the frequency spectrum [Fig. 6(b)]. Increasing the excitation frequency above $\mathrm{St}_{e}$ $\sim 1.1$, an irregular process of vortex pairing is initiated in the jet zone and consequently the frequency of the velocity signal starts to switch between the fundamental and subharmonic [Fig. 5(c)]. The subharmonic peak observed in the spectrum reaches almost the same level as the fundamental peak [Fig. 6(c)]. Its twin-peak character suggests that the subharmonic mode appears in a modulated manner. If the excitation with $\mathrm{St}_{e}>1.45$ is applied, the process of vortex pairing is regular and the velocity signal resumes its periodic character [Fig. 5(d)]. The frequency of velocity fluctuations is then just half of the excitation frequency [Fig. 6(d)]. If the excitation frequency is increased above $\mathrm{St}_{e} \sim 2.1$, the signal periodicity fades away again [Figs. 5(e) and 6(e)]. Finally, for $\mathrm{St}_{e}>3$, the signal and its frequency spectrum reestablish the character observed under unexcited flow conditions [Figs. 5(f) and 6(f)].

The velocity rms values measured under excited flow conditions at the same near-wall location are compared in Fig. 7. In order to separate the coherent (periodic) and broadband (random) components of the velocity fluctuations, the measured signals were bandpass-filtered to contain only the fluctuations corresponding to the excitation frequency $f_{e}$ (circles) or its first subharmonic $f_{e} / 2$ (triangles). The dashed line represents the local magnitude of velocity $\mathrm{rms}\left(u^{\prime} / U\right.$ $=0.22$ ) measured under unexcited flow conditions. The frequency ranges of different flow regimes are also indicated in this figure. Depending on the excitation frequency, the velocity rms value is locally increased or decreased by the jet excitation. At this specific radial location, the maximum
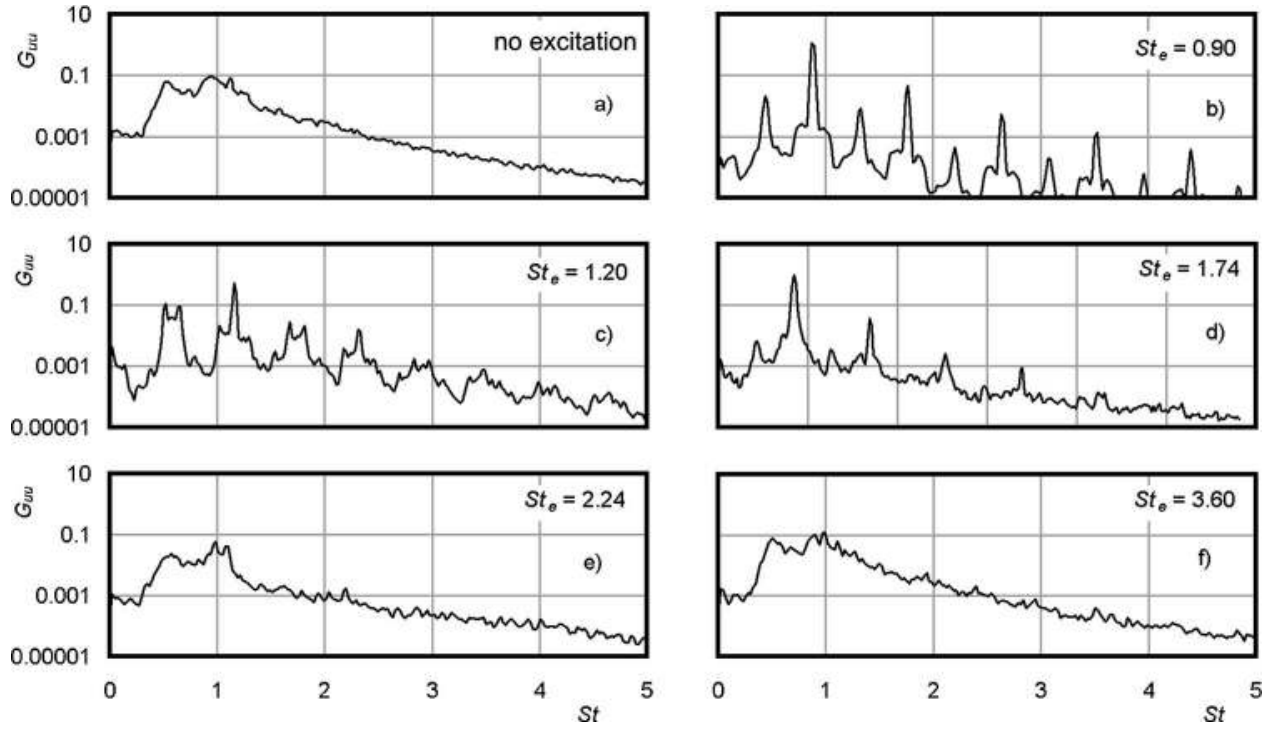

FIG. 6. Power spectra of near-wall velocity fluctuations calculated for data presented in Fig. 5. 


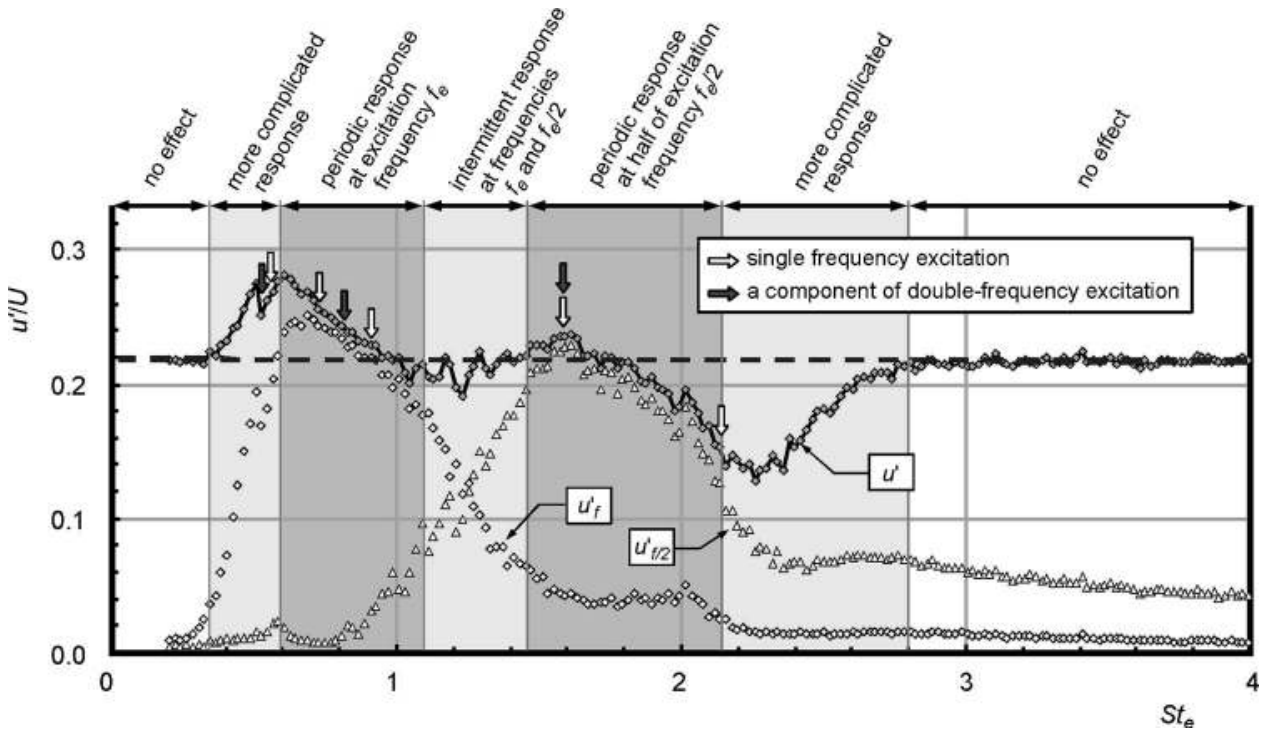

FIG. 7. The different flow regimes identified with respect to the excitation frequency from the near-wall velocity data measured at $r / D=1$ and $y / D$ $=0.04$. The arrows denote the excitation cases investigated in detail in Sec. III B. value of $u^{\prime} / U=0.28$ is achieved at $\mathrm{St}_{e}=0.6$. For the excitation $\mathrm{St}_{e}$ ranging from 0.6 to 1.1 , large regular vortices tend to dominate in the near-wall flow region and therefore the periodic component of the velocity fluctuations prevails over the random component. The mechanism of vortex pairing typical in the subharmonic flow regime ( $\mathrm{St}_{e}$ from 1.45 to 2.1 ) also causes high flow periodicity but lower fluctuation levels (e.g., $u^{\prime} / U=0.16$ at $\mathrm{St}_{e}=2.1$ ). The velocity fluctuations are highly suppressed when the jet is excited at a frequency about $\mathrm{St}_{e} \sim 2.25$. The corresponding shear layer Strouhal number is $\mathrm{St}_{\theta}=0.019$ and thus near $\mathrm{St}_{\theta}=0.017$, the value at which a suppression of velocity fluctuations in free jets were observed. ${ }^{11}$ The velocity signal [Fig. 5(e)] suggests that the flow near the wall consists of small disorganized vortices.

The radial distributions of $u^{\prime} / U$ measured for the selected excitation conditions are presented in Fig. 8. During these measurements the probe was moved along the line $y / D=0.04$ parallel to the wall. The distribution obtained under unexcited flow conditions is characterized by a steep increase behind the stagnation zone with a maximum at the radial position of $r / D \sim 1.1$, then by a gradual decrease at larger radii. When the periodic flow regime is induced by entrance excitation, the maximum value of $u^{\prime} / U$ is enhanced and its location is shifted slightly toward the stagnation

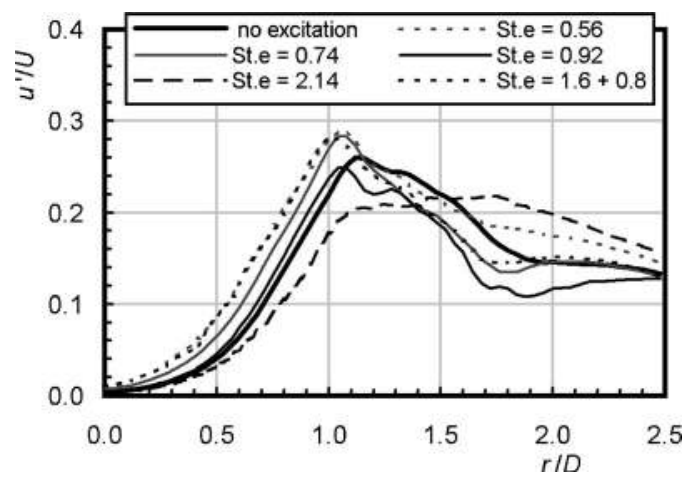

FIG. 8. Effect of the excitation on the radial profile of near-wall velocity rms values measured at $y / D=0.04$. point. This low-frequency excitation leads to an increase of $u^{\prime} / U$ at small radii, but a decrease at large radii. By contrast the opposite local effects are observed at high excitation frequencies (for $\mathrm{St}_{e}$ from 2.1 to 2.5). This feature is linked to the process of unsteady flow separation, which can be either enhanced or suppressed by a specific jet excitation.

The time-average velocity profiles in the wall jet region are shown in Fig. 9(a) for three different jet flow conditions. The modification of the velocity profile by the entrance flow excitation is especially apparent at the radial location $r / D$ $=1.44$. The vortex impingement onto the wall brings about boundary layer separation, ${ }^{25}$ which is accompanied by vortex rebound from the wall. In the separating flow, the velocity maximum moves away from the wall. As the excitation at $\mathrm{St}_{e}=0.74$ leads to large vortices of high circulation, the vortex rebound and separation are more pronounced. ${ }^{26}$ By contrast the excitation at $\mathrm{St}_{e}=2.14$ (irregular flow regime with small vortices) generates a near-wall velocity profile with a maximum located even closer to the wall than in the case of unexcited jet. The moving fluid remains near the wall and any flow separation is prevented at these excitation conditions. The velocity rms profiles corresponding to the same measurement conditions are presented in Fig. 9(b). The inflexion character of the near-wall velocity profile, observed in the case of separating flows $\left(\mathrm{St}_{e}=0.74\right)$, brings about two distinct peaks on the near-wall velocity rms profile. Because of boundary layer separation, its maximum value is progressively shifted away from the wall. The flow configuration of small disorganized eddies $\left(\mathrm{St}_{e}=2.14\right)$, is not able to provoke an unsteady flow separation. The maximum value of velocity rms is lower, but remains close to the wall.

\section{B. Velocity field in the excited impinging jet}

We have seen in the previous section that the velocity signal near the wall is strongly modified by excitations applied to the jet. In order to obtain a more complete picture of impinging jet flow under controlled excitation conditions, detailed measurements of the entire velocity field were carried out and a phase-averaging technique was used to eluci- 

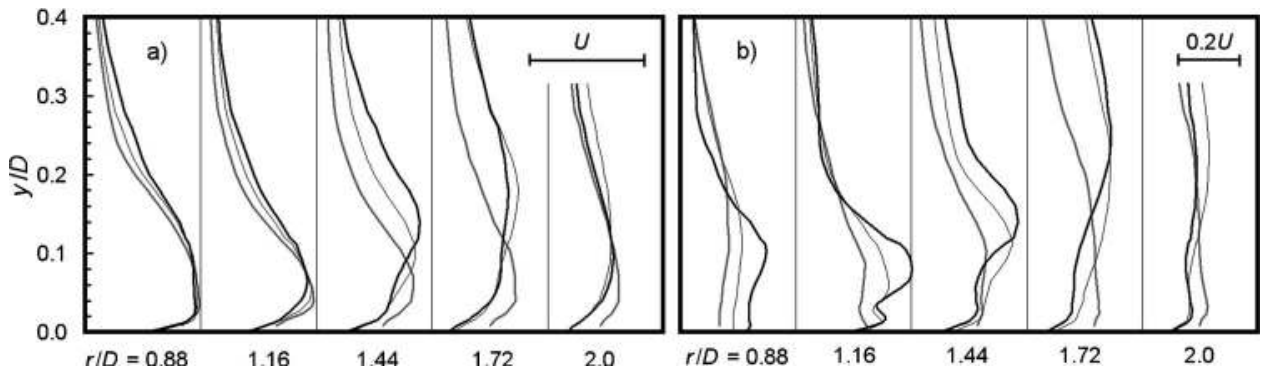

FIG. 9. Effect of jet excitation on the velocity profiles in the wall jet region. (a) time-average velocity and (b) velocity rms (thin black line-unexcited jet, thick black line-jet excited at $\mathrm{St}_{e}=0.74$, thick gray line-jet excited at $\left.\mathrm{St}_{e}=2.14\right)$. date vortex behavior. In the following section we present the results obtained for seven different excitation cases. Their excitation frequencies are marked by arrows in Fig. 7.

\section{Case I: Jet excitation at $\mathrm{St}_{e}=0.74$}

This case with periodic jet flow response is considered representative and demonstrates the evolution of typical flow structures observed in the impinging jet. Two possible presentations of the phase-averaged velocity field (shown in Fig. 10) were constructed from the locally measured velocity signals for a particular phase of the jet excitation period. The typical flow structures which appear regularly in the jet and wall zones are shown in this figure. The second map [Fig. $10(b)]$ is based on the local differences between the phaseaveraged and time-averaged velocities and gives a better picture of the flow structures in the regions where large gradients of the phase-averaged velocity are observed.

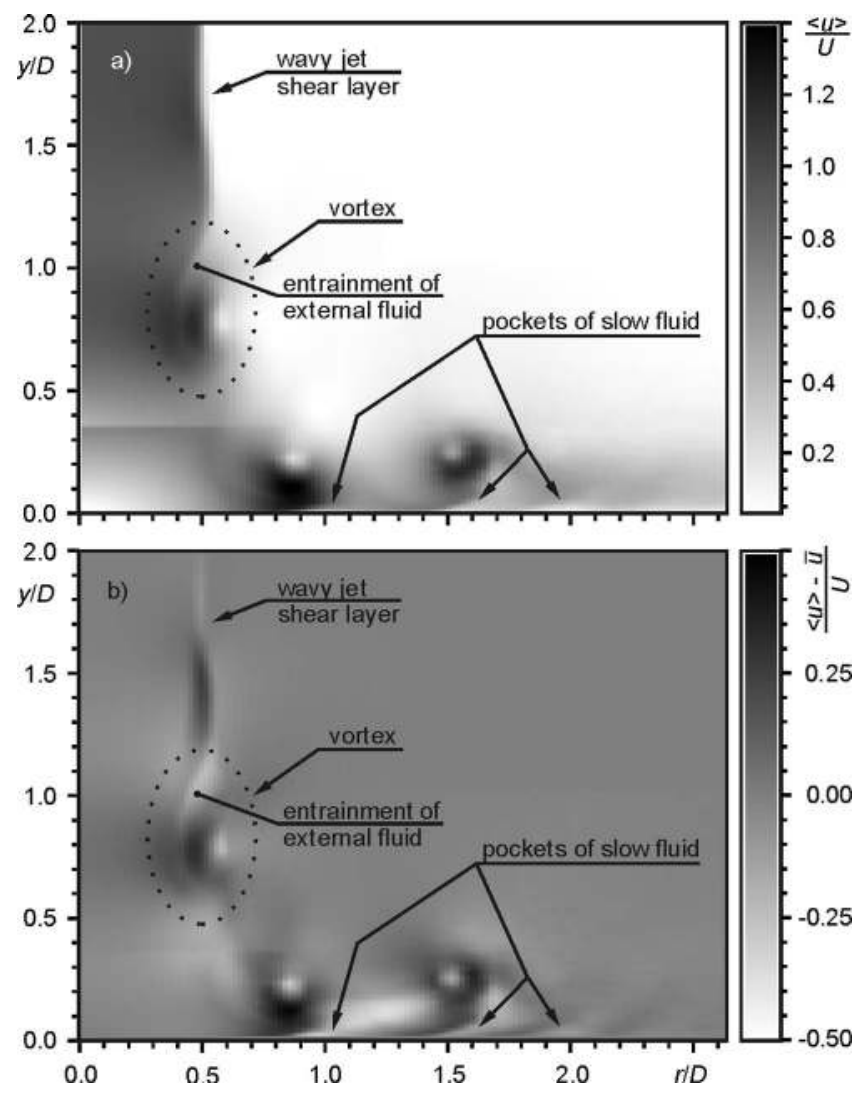

FIG. 10. Velocity fields in the excited impinging jet $\left(\mathrm{St}_{e}=0.74\right)$ : (a) phaseaveraged velocity and (b) difference between the phase-averaged and timeaveraged velocity.
The shear layer waviness is a direct consequence of the jet bulk excitation. Periodic changes in the nozzle exit velocity cause oscillations in the jet diameter resulting in a wavy shear layer. The subsequent processes of vortex roll-up, movement, and impact on the wall are captured in two following figures where five consecutive velocity fields illustrate the flow evolution during one excitation period in more detail.

The vortex rolling-up controlled by the jet excitation at $\mathrm{St}_{e}=0.74$ is shown in Fig. 11. This process is accomplished at a distance less than 1D from the nozzle exit. The velocity is increased on the high-speed side of the shear layer and decreased on its low-speed side. The local variation of phaseaveraged velocity reaches about $30 \%$ of the average velocity at the nozzle exit [see the grade scale in Fig. 11(b)]. The pairs of fast and slowly moving fluid bodies grow and finally form vortices. The counterclockwise rotation of fluid inside the vortices can be deduced from the velocity field presented in Fig. 11. The vortex motion entrains the surrounding fluid into the jet and a wedge shaped body of slowly moving fluid (visible in the last frame of the velocity field sequence) splits the body of faster fluid. The on-screen animation reveals that the body of slowly-moving fluid is wrapped completely around the vortex and it splits the lump of the fast fluid once more (indistinctly seen in the first frame when the bottom vortex is focused). The fluid entrainment is accompanied by a deformation and enlargement of the shear layer between the jet and the surrounding fluid.

The time sequence of flow maps in Fig. 12 shows the impact of vortices on the wall and their subsequent rebound. A remarkable feature of these near-wall velocity fields is the presence of "pockets" of slowly moving fluid beneath the vortices. These pockets start to be visible at $r / D \sim 0.9$ (i.e., at the location where the vortices approach the wall) and then grow during their movement along the wall. They are a sign of downstream movement of the unsteady flow separation. According to Didden and Ho, ${ }^{25}$ they are a consequence of the boundary layer deceleration caused by the unfavorable pressure gradient which is imposed by the passing vortex. The fluid above and to the left of these pockets is accelerated by the vortex circulation. As the vortices rebound from the wall, the regions of fast fluid leave the wall. As our measurements are not direction-sensitive, the formation of a secondary vortex rotating in opposite direction cannot be detected. However, the flow structure could be interpreted as shown in the second frame of Fig. 12. This interpretation is in accordance with other studies. ${ }^{20,25,26}$ The pairs of vortices then depart from the wall due to the mutual interaction between 

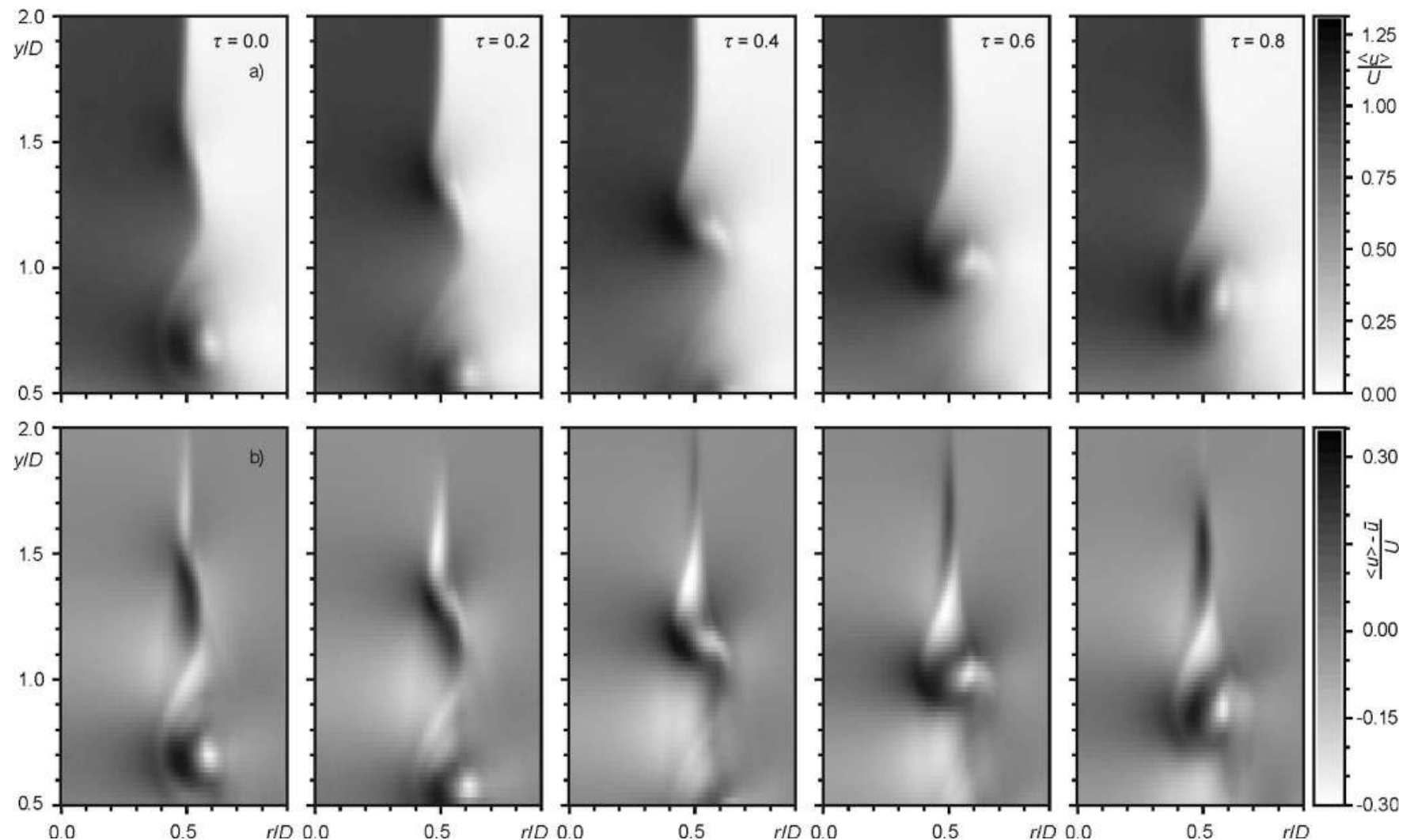

FIG. 11. Evolution of the phase-averaged velocity field in the excited jet zone $\left(\mathrm{St}_{e}=0.74\right)$ : (a) phase-averaged velocity and (b) difference between phaseaveraged and time-averaged velocity. $\tau=t f_{e}$ is a particular phase of the excitation period.

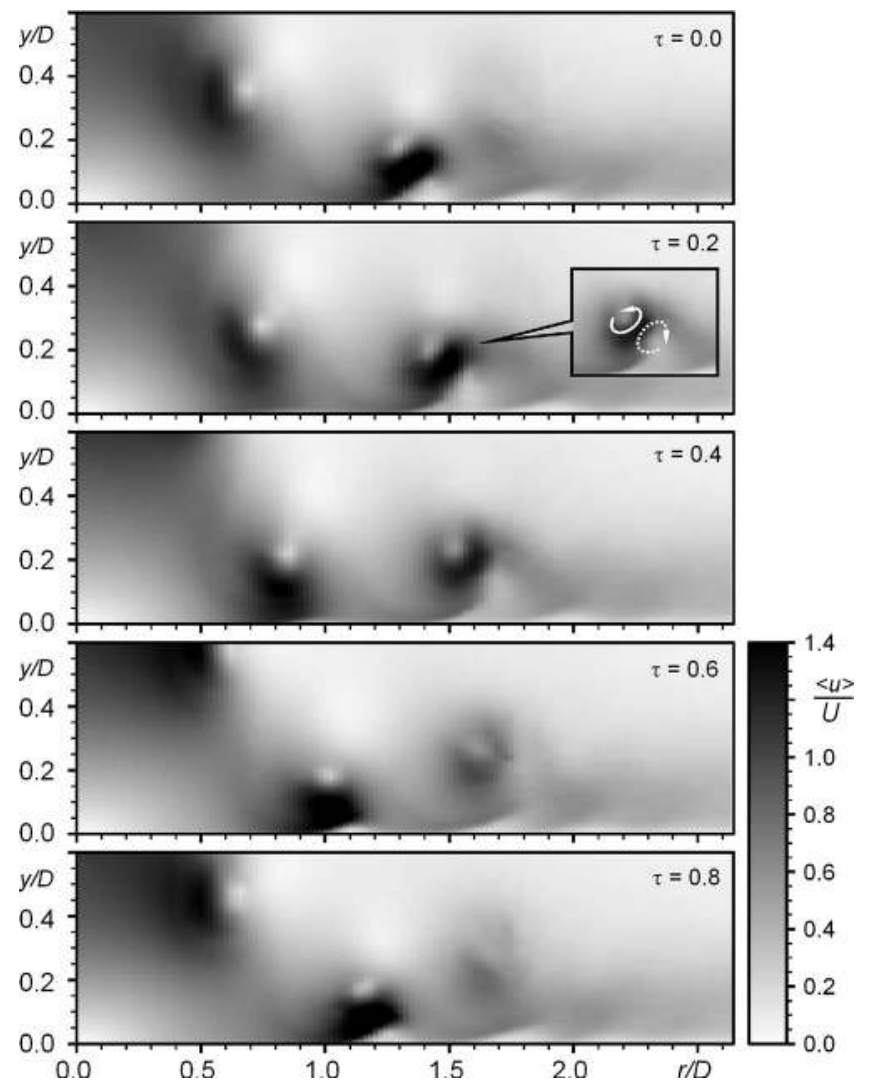

FIG. 12. Evolution of the phase-averaged velocity field in the wall jet region of the excited jet $\left(\mathrm{St}_{e}=0.74\right)$. the primary and secondary vortex. The vortex movement can also explain why the velocity maximum leaves the wall at radii $r / D>1$ (see Figs. 9 and 15).

After rolling-up in the shear layer, the vortices are dragged by the mean flow along the trajectory which is indicated in Fig. 13. The instantaneous position of the center of the vortex is here rather arbitrary determined as the location, where the velocity gradient is locally largest. The phaseaveraging technique is able to follow the vortex movement until it reaches a certain radial position. For the given excitation conditions, the vortex becomes blurred at a radius of

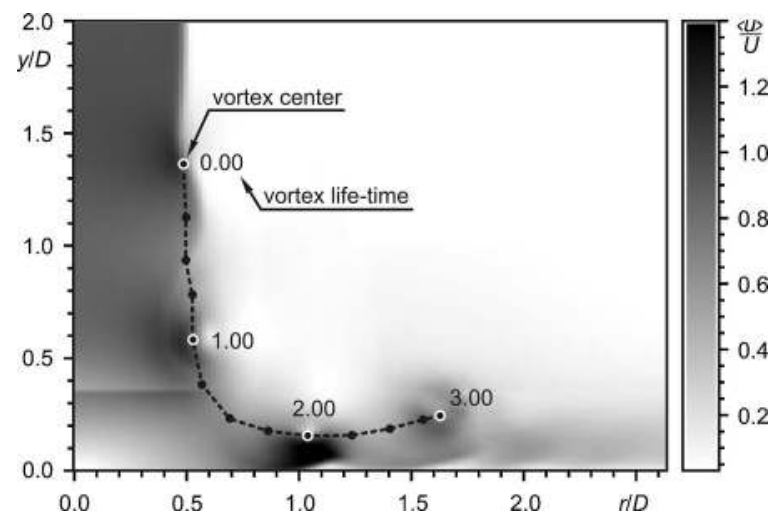

FIG. 13. Vortex trajectory inside the excited impinging jet $\left(\mathrm{St}_{e}=0.74\right)$ determined from a sequence of phase-averaged velocity fields. Vortex lifetime is expressed as a number of excitation periods elapsed from the vortex formation. 


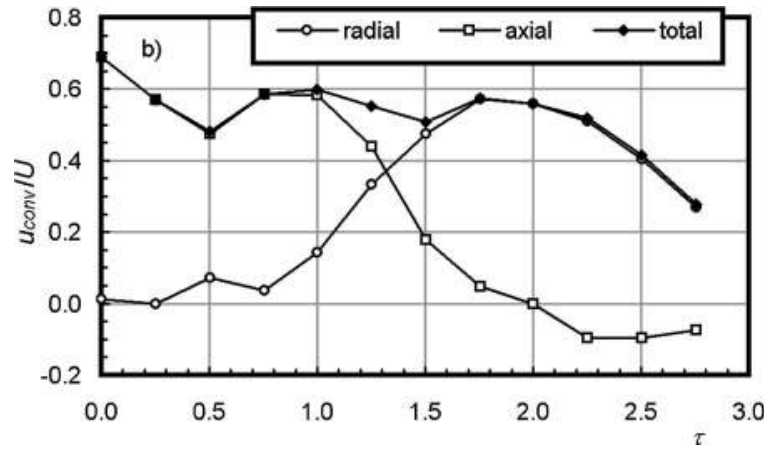

FIG. 14. Vortex convection velocity as a function of the vortex lifetime in the excited impinging jet $\left(\mathrm{St}_{e}=0.74\right)$. The axial velocity component has an inverted sign with positive values for the movement toward the wall.

$r / D \sim 1.7$ where the vortex lifetime reaches approximately three excitation periods. We cannot determine from our experiments whether the vortices disappear from the phaseaveraged velocity field due to the loss of phase-locking with the excitation, vortex breakup or three-dimensional effects. From the vortex trajectory, the convection velocity of vortices can be estimated. This velocity is shown in Fig. 14 as a function of the vortex lifetime (measured in the number of excitation periods). Its absolute magnitude varies around a value of $0.55 U$, which agrees with the results of previous measurements performed in a free jet configuration. ${ }^{6}$ The vortex slows down and loses about $50 \%$ of its convection velocity during the third phase period, which takes place after the interaction of the vortex with the wall.

Time-averaged velocity maps are presented in Fig. 15. The vortex trajectory has a footprint in the locally increased
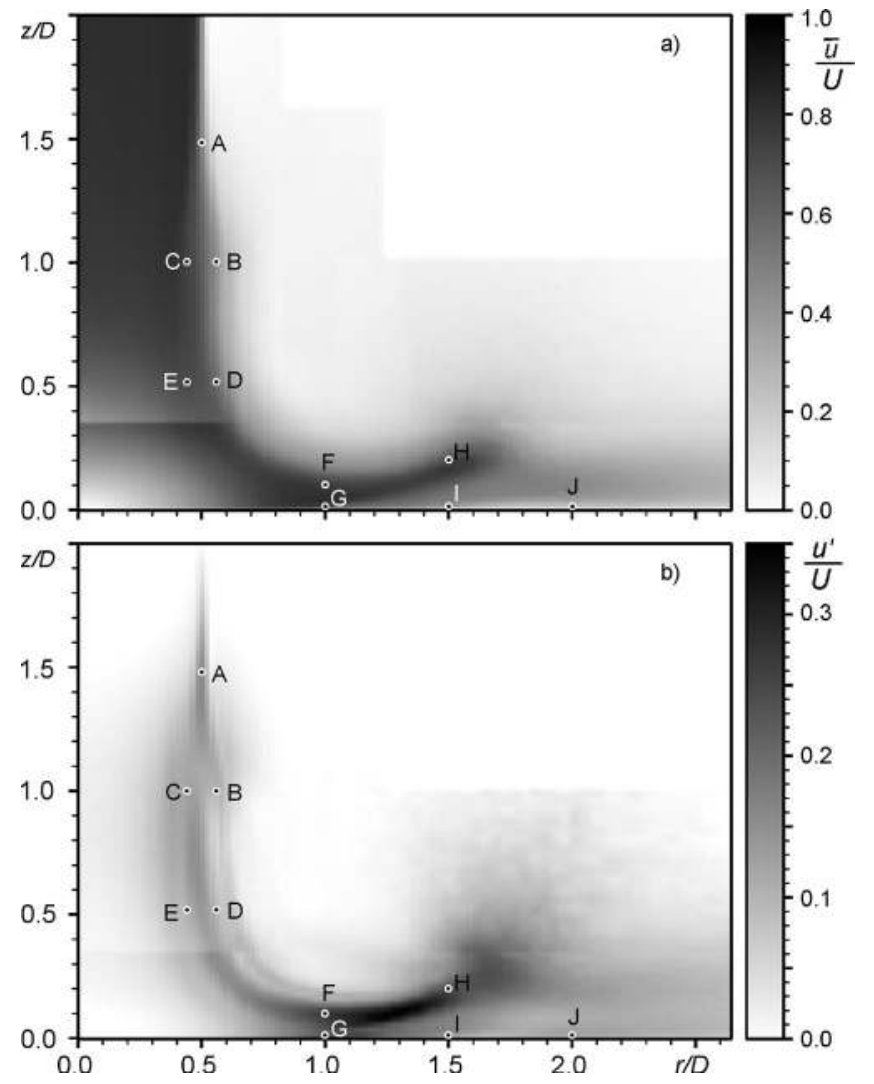

FIG. 15. Time-averaged velocity fields for the excited impinging jet $\left(\mathrm{St}_{e}\right.$ $=0.74$ ): (a) time-averaged velocity and (b) velocity rms.

values of the velocity rms. The velocity maximum gradually departs from the wall (at radial locations $r / D>1.1$ ) due to the unsteady flow separation. The time-averaged velocity field of an unexcited jet (not shown here, but available on the
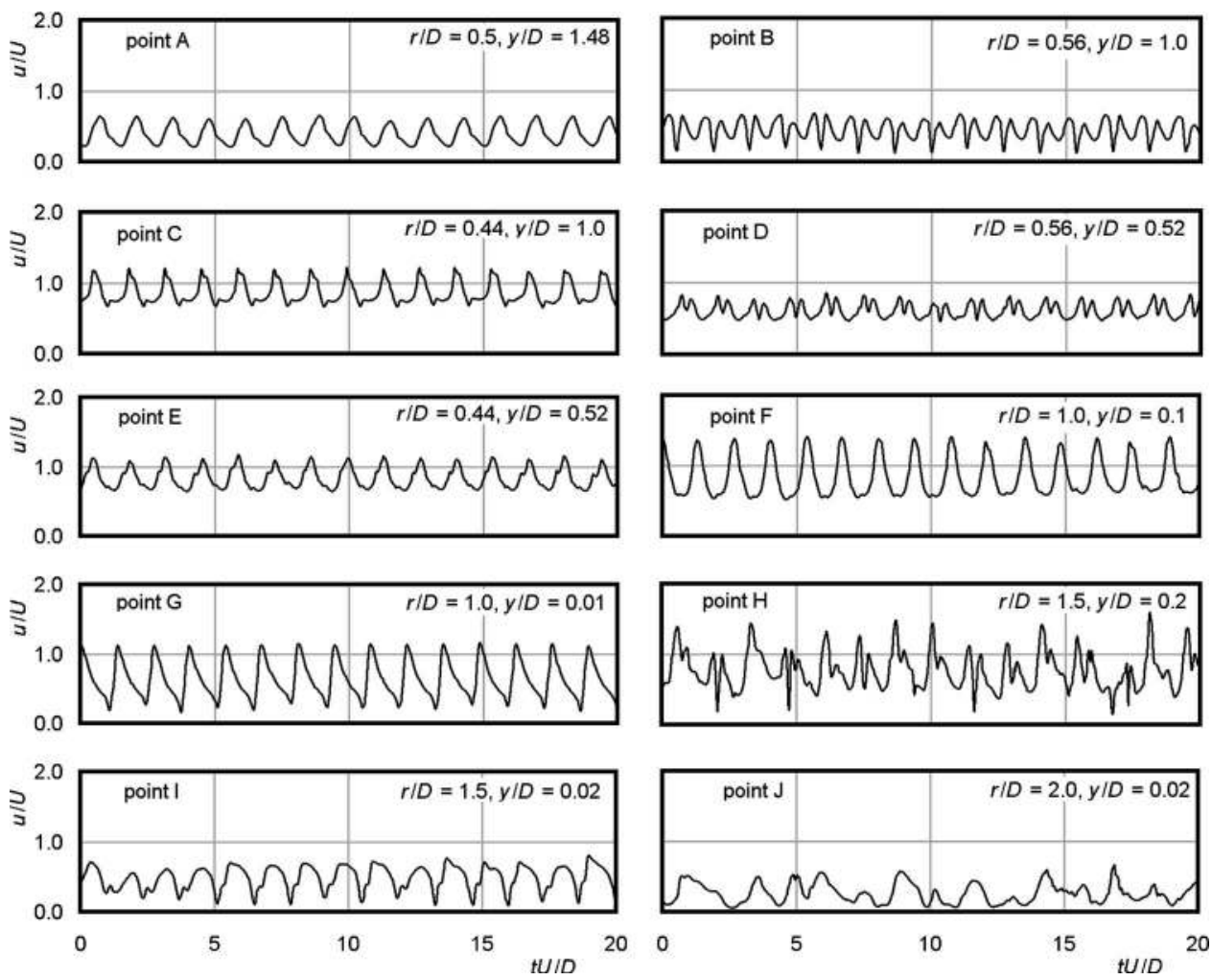

FIG. 16. Velocity signals measured at different locations inside the excited impinging jet $\left(\mathrm{St}_{e}=0.74\right)$. See Fig. 15 for the point's localization. 


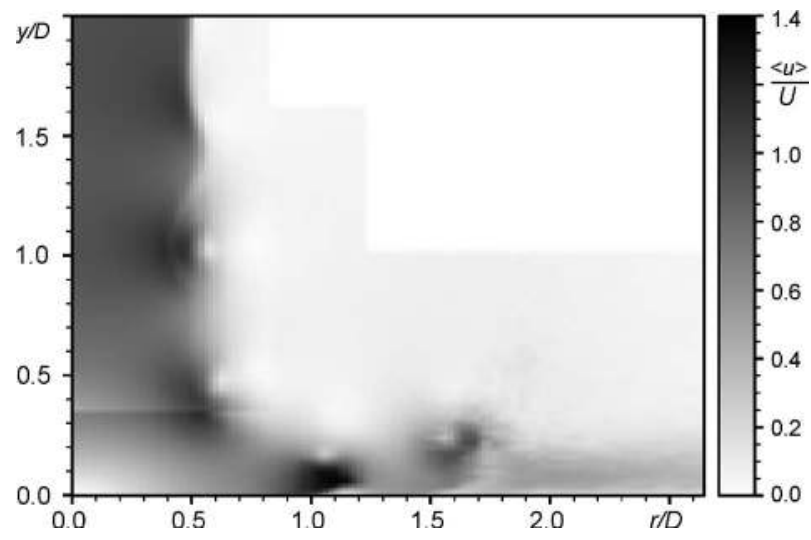

FIG. 17. Phase-averaged velocity fields in a jet excited at $\mathrm{St}_{e}=0.92$.

EPAPS site $^{30}$ ) has a similar character, suggesting that the unsteady flow separation also occurs in unexcited jets.

The labeled points in Fig. 15 mark the specific locations, for which the velocity time series are presented in Fig. 16. Some links between the character of velocity signals and the structure of the phase-averaged velocity fields can be identified. The oscillations of the velocity in point A are caused by the waviness of the shear layer, which causes the anemometer probe to emerge alternately on either the high-speed or the low-speed side of the shear layer. The sharp narrow valleys in the signals measured in points B and D correspond to the passage of the small bulks of slowly moving fluid, which are presented near the vortex centers (see Fig. 11). The short and weak decrease of velocity near the peaks and valleys, apparent at points $\mathrm{C}$ and $\mathrm{E}$, can be attributed to the passage of the wedge body of the slowly moving fluid. The velocity signal loses periodicity at $r / D \sim 1.5$, but the signal remains periodic slightly longer in the near wall region (compare points $\mathrm{H}$ and $\mathrm{I}$ ).

\section{Case II: Jet excitation at $\mathrm{St}_{e}=0.92$}

When the excitation frequency is increased to $\mathrm{St}_{e}=0.92$, the impinging jet is still in the periodic response regime. The shear layer starts to wave sooner than in the previous case and with a shorter wavelength. The vortex rolling-up process is more rapid and the shear layer rapidly becomes thick. As

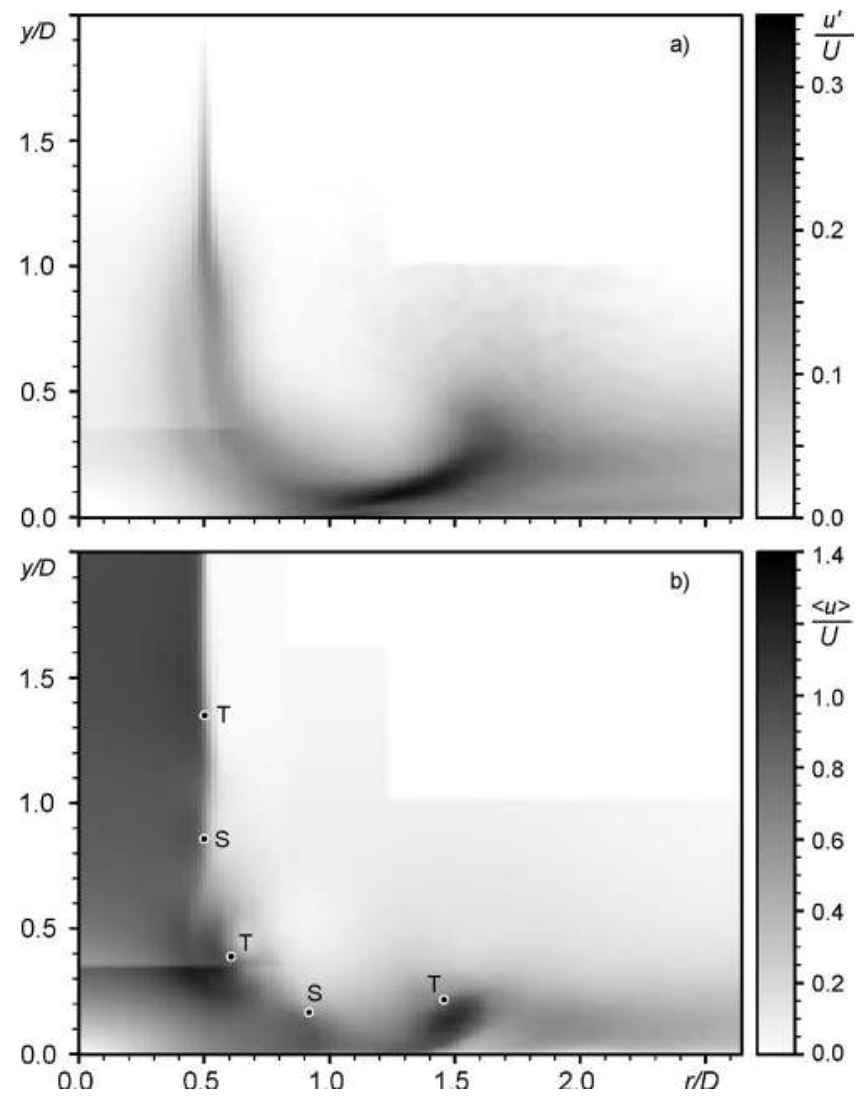

FIG. 18. Velocity fields of the excited impinging jet $\left(\mathrm{St}_{e}=0.56\right)$ : (a) velocity rms and (b) phase-averaged velocity.

seen in Fig. 17, the character of the phased-averaged velocity field is preserved, only the spacing between vortices becomes shorter. The vortex survival time reaches four excitation periods, which is the greatest number of periods we have observed. Compared to the other investigated cases, the periodic nature of the flow was preserved in most of the measuring domain.

\section{Case III: Jet excitation at $\mathrm{St}_{e}=0.56$}

The excitation frequency corresponding to $\mathrm{St}_{e}=0.56$ is slightly below the range in which the jet response is periodic. Fig. 18 shows velocity field for this excitation case. The
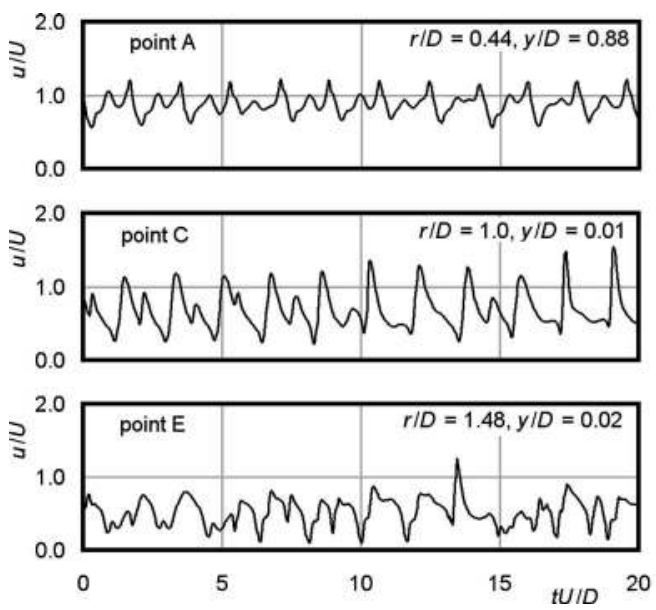
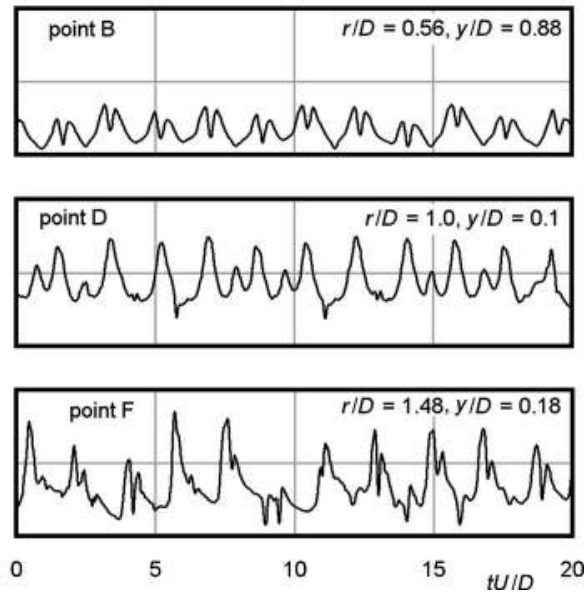

FIG. 19. Velocity signals measured at the different locations inside the excited impinging jet $\left(\mathrm{St}_{e}=0.56\right)$. 


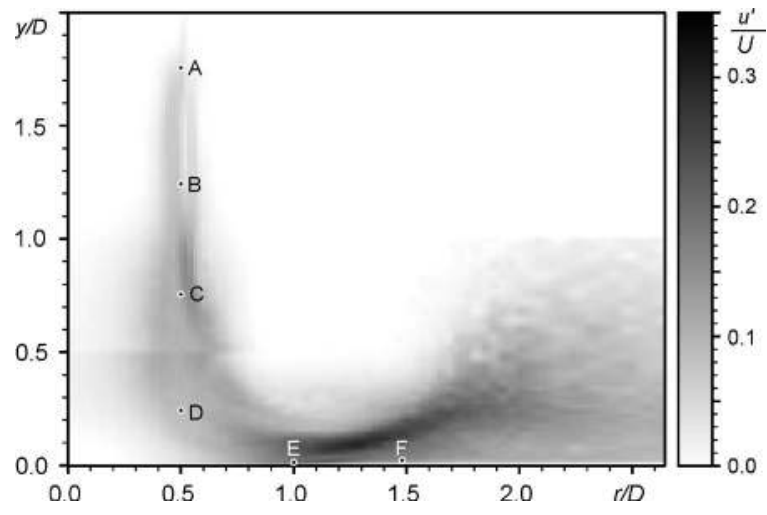

FIG. 20. Velocity rms in the impinging jet excited at $\mathrm{St}_{e}=1.60$.

shear layer waviness is not as pronounced as in previous cases and the vortex rolling-up occurs farther downstream from the nozzle. The phase-averaged velocity field reveals two types of vortices. The formation of the vortices, marked with the letter "T," is triggered by the excitation, whereas the vortices denoted by "S" form spontaneously because the distance separating the $\mathrm{T}$ vortices is too great to provide a stable shear layer. The phase jitter together with relative weakness of $\mathrm{S}$ vortices makes them hardly visible in the phaseaveraged velocity field. The velocity signals shown in Figs. 19(a) and 19(c) exhibit large and relatively regular fluctuations separated by an interval of $1 / \mathrm{St}_{e}=1.79$ as well as small and irregular ones appearing meanwhile. The former indicates the passage of $\mathrm{T}$ vortex whereas the other is evidence of the appearance of $\mathrm{S}$ vortex at the probe. The pockets of slowly moving fluid are still present beneath all the vortices. However, there is a lot of irregularity in the velocity signals measured near the wall [Figs. 19(c) and 19(e)].

\section{Case IV: Jet excitation at $\mathrm{St}_{e}=1.6$}

For the excitation frequency corresponding to $\mathrm{St}_{e}$ from 1.45 to 2.1 , a subharmonic response is observed in the nearwall flow region of the impinging jet. The field of the velocity rms for an excitation of $\mathrm{St}_{e}=1.6$ is shown in Fig. 20. The shear layer thickening proceeds in two distinct steps: the first time about the point $\mathrm{A}$ and the second time about halfway between the points B and C. This is the location where pairs

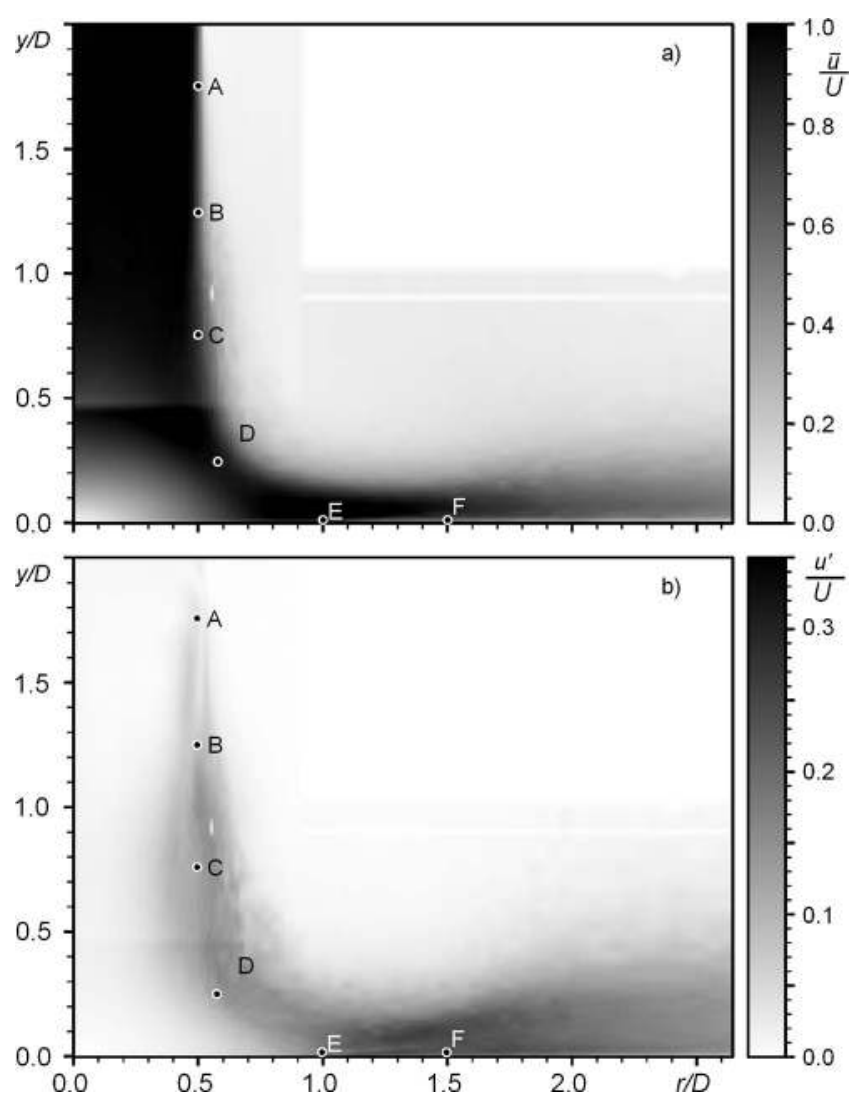

FIG. 22. Time-averaged velocity fields for the excited impinging jet $\left(\mathrm{St}_{e}\right.$ =2.14): (a) time-averaged velocity and (b) velocity rms.

of subsequent vortices merge to create larger and stronger vortices passing at the half frequency $f_{e} / 2$. As the reference signal used for phase averaging is the excitation signal, the phase averaging cannot reveal the instantaneous flow structure downstream the vortex merging. The frequency halving is clearly visible in Fig. 21, where the velocity signals recorded along the vortex trajectory are presented. The location of vortex pairing is not stationary, but moves upstream and downstream, as is shown by the modulated character of the velocity signal measured at the point $\mathrm{B}$.
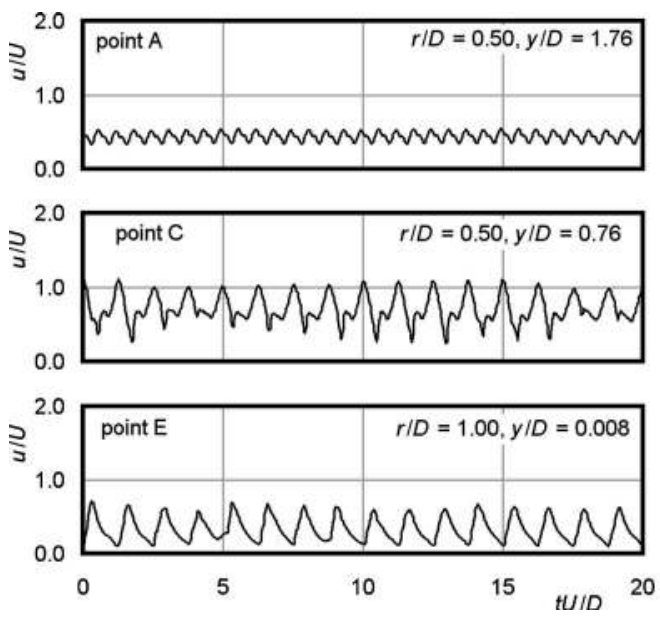
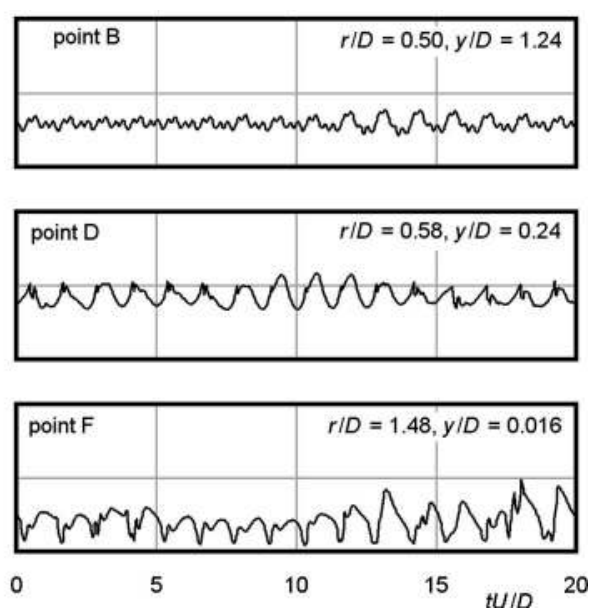

FIG. 21. Velocity signals measured at the different locations inside the excited impinging jet $\left(\mathrm{St}_{e}=1.6\right)$. See Fig. 20 for the measuring point's localization. 

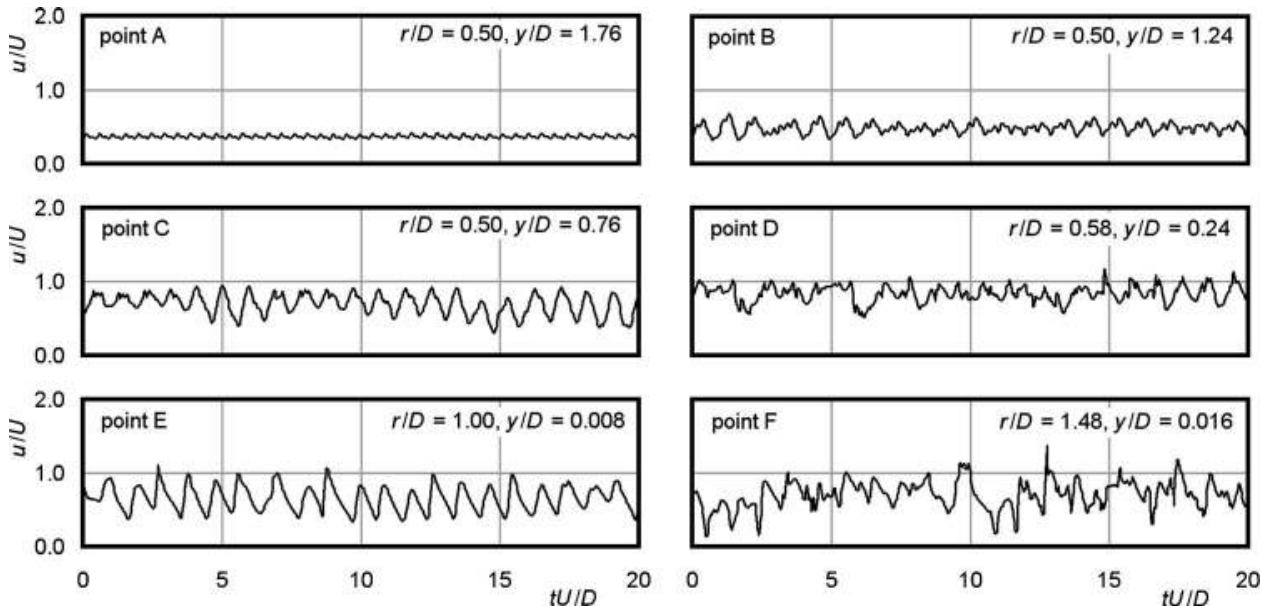

FIG. 23. Velocity signals measured at different locations inside the excited impinging jet $\left(\mathrm{St}_{e}=2.14\right)$. See Fig. 22 for the point's localization.

\section{Case V: Jet excitation at $\mathrm{St}_{e}=\mathbf{2 . 1 4}$}

The jet excitation at the frequency corresponding to the shear-layer Strouhal number $\mathrm{St}_{\theta} \sim 0.017$ initially leads to a rapid wave growth in the shear layer. However the wave growth saturates at low amplitude and the shear layer contains small and relatively stable vortices. The velocity fluctuations in the jet are then suppressed. ${ }^{11}$ This flow regime was observed at $\mathrm{St}_{e}=2.14$ which corresponds in the shear layer thickness scaling to the value of $\mathrm{St}_{\theta}=0.018$. The nearwall velocity fluctuations at the vortex impact location were greatly attenuated (see Fig. 7). The circulation of small vortices is not strong enough to trigger the unsteady flow separation and consequently the velocity field presented in Fig. 22 differs remarkably from those observed in the other investigated cases (e.g., compare with those shown in Fig. 15). The jet of high-speed fluid remains in the near-wall region and the velocity maximum does not depart from the wall (see Fig. 9). Thus the near-wall velocity fluctuations at larger radii are stronger than in the unexcited impinging jet (see Fig. 8). The velocity signals shown in Fig. 23 demonstrate that the velocity fluctuations are relatively small and are modulated or nonperiodic. This qualitatively different flow regime is of particular interest for studies into the control of the heat transfer between a wall and an impinging jet.

\section{Case VI: Jet excitation simultaneously at $\mathrm{St}_{e}=1.60$ and 0.80}

To enable the application of phase-averaging technique in the cases when vortex pairing occurs, the jet was simultaneously excited at two frequencies: the fundamental $\mathrm{St}_{e}$ $=1.60$ and its subharmonic $\mathrm{St}_{e} / 2=0.8$. In this case, the subharmonic excitation signal served as a suitable phasereference for phase-averaging. The velocity field observed in the case of monochromatic excitation with $\mathrm{St}_{e}=1.60$ (see Fig. 20) was markedly modified by the presence of the slight subharmonic excitation, whose amplitude was chosen to be three times smaller than the fundamental. The phaseaveraged velocity field in Fig. 24 shows the evolution of vortex structures during one subharmonic period. The subharmonic excitation produces variations in the size, strength, and convection velocity of the individual vortices. The process of vortex pairing accelerates, becomes regular and runs over in accordance to the description suggested by Hussain and Zaman. ${ }^{16}$ When the vortex pairing is completed, the near-wall flow field is qualitatively the same as that observed in the cases where $\mathrm{St}_{e}=0.74$ or 0.92 .
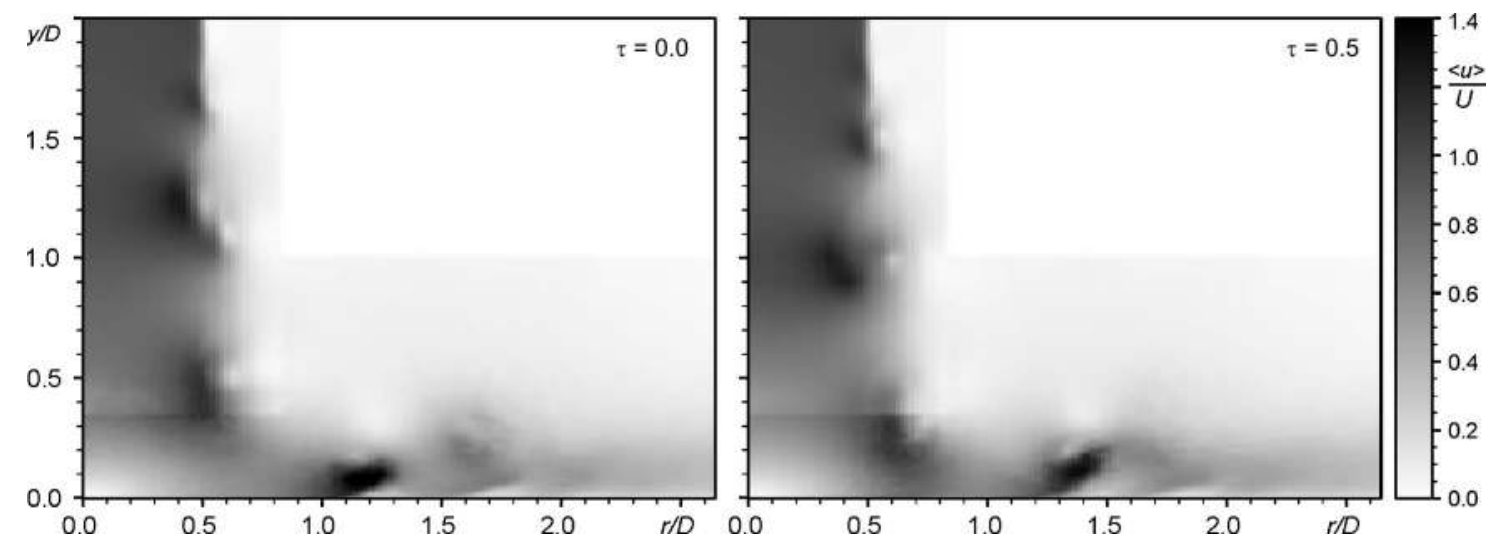

FIG. 24. Time sequence of the phase-averaged velocity fields for two-frequency excitation $\left(\mathrm{St}_{e}=1.60\right.$ and 0.80$)$. 

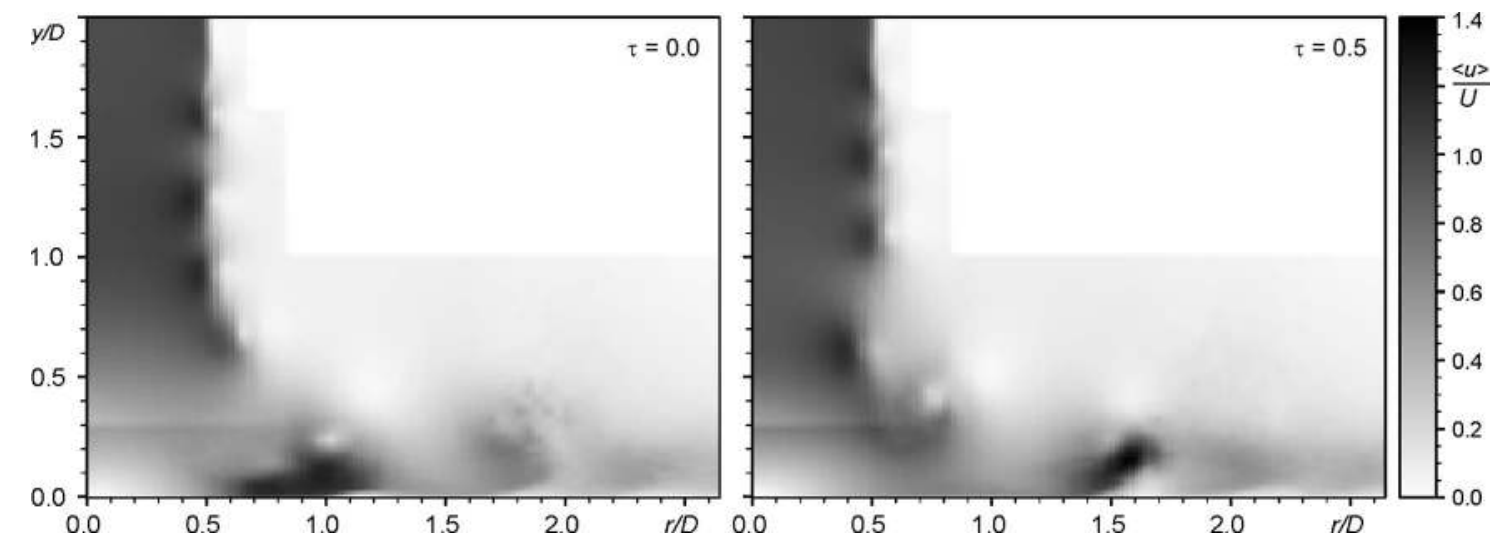

FIG. 25. Time sequence of the phase-averaged velocity fields of a jet excited at two frequencies $\left(\mathrm{St}_{e}=1.60\right.$ and 0.53$)$.

\section{Case VII: Jet excitation simultaneously at $\mathrm{St}_{e}=1.60$ and 0.53}

The jet excited at $\mathrm{St}_{e}=1.60$ is also sensitive to the second subharmonic frequency $\mathrm{St}_{e} / 3=0.53$. The vortex rolling-up is again controlled by the fundamental excitation, but now the two-frequency excitation leads to the merging of three vortices. The vortex triplets merge into large vortices, which travel down and hit the wall with the frequency prescribed by the subharmonic excitation. Although the excitation level at $\mathrm{St}_{e}=0.53$ is three times weaker than in case III (monochromatic excitation at $\mathrm{St}_{e}=0.56$ ), the resulting near-wall velocity field is much more periodic. Such a process of vortex triplet merging is visible in Fig. 25.

As the two-frequency excitation is prescribed by five parameters (two frequencies, two amplitudes, and the phase shift between two excitation signals), its final effect on the jet velocity field is expected to be more complicated than was illustrated in the two previous cases. According to Hussain and Hussain, ${ }^{12}$ the phase shift, $\varphi$, is the parameter which controls the process of vortex pairing in the case of subharmonic excitation. In our preliminary test, we did not observed any significant dependence on $\varphi$. However, these test measurements were not detailed and a narrow band of the phase shift, in which vortex pairing could be strongly sensitive to $\varphi$, may have been omitted.

\section{CONCLUSIONS}

The effect of an external excitation on circular impinging jet flow was documented. The flow was found to be sensitive to excitations in a wide frequency range. Different flow regimes were identified in the near-wall region depending on the excitation frequency: a periodic regime exhibiting flow fluctuations locked on the excitation frequency $f_{e}$, a regime irregularly alternating between the fundamental frequency $f_{e}$ and the subharmonic frequency $f_{e} / 2$, a subharmonic periodic regime with regular vortex pairing and finally border regimes with complicated nonperiodic flow response.

The near-wall velocity fluctuations can be either intensified or attenuated by the excitation. The resulting effect depends on the strength of vortices arriving at the wall. The regimes with periodic behavior produce large vortices, which after approaching the wall locally increase the near-wall flow fluctuations and then provoke an unsteady flow separation. The maxima on time-average and rms velocity profiles move away from the wall downstream of this separation. By contrast, vortices produced by the excitation at frequencies near $\mathrm{St}_{\theta} \sim 0.017$ are small and consequently, the flow fluctuations around their impingement are attenuated. These small vortices are unable to trigger the flow separation and thus the fluid stream remains attached to the wall. The results of twofrequency excitation experiments confirm that the flow is sensitive to the subharmonic excitation. This method permits the excitation of some low-frequency flow regimes which cannot be achieved by monochromatic excitation.

The phase-averaging technique provided some insight into the behavior of vortex structures inside the impinging jet. The processes taking place during the vortex impact on the wall and the subsequent unsteady flow separation were demonstrated. However, the question of how the changes in the near-wall velocity field, which are caused by the jet excitation, affect heat and mass transfer processes at the wall remains unanswered.

\section{ACKNOWLEDGMENTS}

This research was supported by the Academy of Sciences of the Czech Republic (Project No. K4040110) and by the European Commission (Project No. G1MA-CT-200204019).

${ }^{1}$ B. W. Webb and C. F. Ma, "Single-phase liquid jet impingement heat transfer," Adv. Heat Transfer 26, 105 (1995).

${ }^{2}$ A. Michalke, "On the spatially growing disturbances in an inviscid shear layer," J. Fluid Mech. 23, 521 (1965).

${ }^{3}$ G. E. Mattingly and C. C. Chang, "Unstable waves on an axisymmetric jet column,” J. Fluid Mech. 65, 541 (1974).

${ }^{4}$ R. E. Drubka, P. Reisenthel, and H. M. Nagib, "The dynamic of low initial disturbance turbulent jets," Phys. Fluids A 1, 1723 (1989).

${ }^{5}$ T. C. Corke, F. Shakib, and H. M. Nagib, "Mode selection and resonant phase locking in unstable axisymmetric jets," J. Fluid Mech. 223, 253 (1991).

${ }^{6}$ A. K. M. F. Hussain and K. M. B. Q. Zaman, "The 'preferred mode' of the axisymmetric jet," J. Fluid Mech. 110, 39 (1981).

${ }^{7}$ D. Liepman and M. Gharib, "The role of streamwise vorticity in the nearfield entrainment of round jets," J. Fluid Mech. 245, 643 (1992).

${ }^{8}$ P. Brancher, J. M. Chomaz, and P. Huerre, "Direct numerical simulation of round jets: Vortex induction and side jets," Phys. Fluids 6, 1768 (1994).

${ }^{9}$ S. C. Crow and F. H. Champagne, "Orderly structure in jet turbulence," J. Fluid Mech. 48, 547 (1971). 
${ }^{10}$ C.-M. Ho and P. Huerre, "Perturbed free shear layers," Annu. Rev. Fluid Mech. 16, 356 (1984).

${ }^{11}$ K. B. M. Q. Zaman and A. K. M. F. Hussain, "Turbulence suppression in free shear flows by controlled excitation," J. Fluid Mech. 103, 133 (1981).

${ }^{12}$ H. S. Hussain and F. Hussain, "Experiments on subharmonic resonance in a shear layer," J. Fluid Mech. 304, 343 (1995).

${ }^{13}$ C.-M. Ho and L.-S. Huang, "Subharmonics and vortex merging in mixing layers," J. Fluid Mech. 119, 443 (1982).

${ }^{14}$ K. B. M. Q. Zaman and A. K. M. F. Hussain, "Vortex pairing in a circular jet under controlled excitation. Part 1. General jet response," J. Fluid Mech. 101, 449 (1980).

${ }^{15}$ G. Broze and F. Hussain, "Nonlinear dynamics of forced transitional jets: Periodic and chaotic attractors," J. Fluid Mech. 263, 93 (1994).

${ }^{16}$ A. K. M. F. Hussain and K. B. M. Q. Zaman, "Vortex pairing in a circular jet under controlled excitation. Part 2. Coherent structure dynamics," J. Fluid Mech. 101, 493 (1980).

${ }^{17}$ T. C. Corke and S. M. Kusek, "Resonance in axisymmetric jets with controlled helical-mode input," J. Fluid Mech. 249, 307 (1993).

${ }^{18}$ K. Jambunathan, E. Lai, and M. A. Moss, and B. L. Button, "A review of heat transfer data for single circular jet impingement," Int. J. Heat Fluid Flow 13, 106 (1992).

${ }^{19}$ C. O. Popiel and O. Trass, "Visualization of a free and impinging round jet," Exp. Therm. Fluid Sci. 4, 253 (1991).

${ }^{20}$ C. C. Landreth and R. J. Adrian, "Impinging of a low Reynolds number turbulent circular jet onto a flat plate at normal incidence," Exp. Fluids 9, 74 (1990).
${ }^{21}$ R. Gardon and J. C. Akfirat, "The role of turbulence in determining the heat transfer characteristics of impinging jets," Int. J. Heat Mass Transfer 8, 1261 (1965).

${ }^{22}$ S. D. Hwang and H. H. Cho, "Effect of acoustic excitation positions on heat transfer and flow in axisymmetric impinging jet: Main jet excitation and shear layer excitation," Int. J. Heat Fluid Flow 24, 199 (2003).

${ }^{23}$ S. V. Alekseenko, D. M. Markovich, and V. I. Semenov, "Effect of external disturbances on the impinging jet structure," Exp. Heat Transfer 6, 109 (1997).

${ }^{24}$ T. Liu and J. P. Sullivan, "Heat transfer and flow structures in an excited circular impinging jet," Int. J. Heat Mass Transfer 39, 3695 (1996).

${ }^{25}$ N. Didden and C. M. Ho, "Unsteady separation in the boundary layer produced by an impinging jet," J. Fluid Mech. 160, 235 (1985).

${ }^{26}$ J. D. A. Walker, C. R. Smith, and A. W. Cerra, and T. L. Doligalski, "The impact of a vortex ring on a wall," J. Fluid Mech. 181, 99 (1987).

${ }^{27}$ C.-M. Ho and N. S. Nosseir, "Dynamics of an impinging jet. Part 1 . The feedback phenomenon," J. Fluid Mech. 105, 119 (1981).

${ }^{28}$ A. M. Naguib and M. M. Koochesfahani, "On wall-pressure sources associated with the unsteady separation in a vortex-ring/wall interaction," Phys. Fluids 16, 2613 (2004).

${ }^{29}$ J. Vejrazka, "Experimental study of a pulsating round impinging jet," Ph.D. thesis, Institut National Polytechnique de Grenoble (2002).

${ }^{30}$ See EPAPS Document No. E-PHFLE6-17-029510 for all measured velocity maps and their animations. This document can be reached via a direct link in the online article's HTML reference section or via the EPAPS homepage (http://www.aip.org/pubserv/epaps.html). 